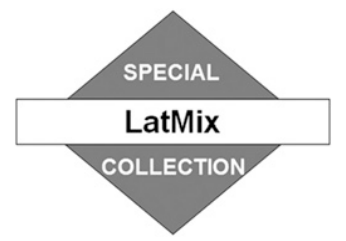

\title{
Dispersion in the Open Ocean Seasonal Pycnocline at Scales of 1-10 km and 1-6 days
}

\author{
Miles A. Sundermeyer AND DANiel A. BIRCH ${ }^{\mathrm{a}}$ \\ University of Massachusetts Dartmouth, North Dartmouth, Massachusetts
}

JAMES R. LEDWELL

Woods Hole Oceanographic Institution, Woods Hole, Massachusetts

\author{
Murray D. Levine, ${ }^{\mathrm{b}}$ Stephen D. Pierce, And Brandy T. Kuebel Cervantes \\ Oregon State University, Corvallis, Oregon
}

(Manuscript received 23 January 2019, in final form 14 November 2019)

\begin{abstract}
Results are presented from two dye release experiments conducted in the seasonal thermocline of the Sargasso Sea, one in a region of low horizontal strain rate $\left(\sim 10^{-6} \mathrm{~s}^{-1}\right)$, the second in a region of intermediate horizontal strain rate $\left(\sim 10^{-5} \mathrm{~s}^{-1}\right)$. Both experiments lasted $\sim 6$ days, covering spatial scales of $1-10$ and $1-50 \mathrm{~km}$ for the low and intermediate strain rate regimes, respectively. Diapycnal diffusivities estimated from the two experiments were $\kappa_{z}=(2-5) \times 10^{-6} \mathrm{~m}^{2} \mathrm{~s}^{-1}$, while isopycnal diffusivities were $\kappa_{H}=(0.2-3) \mathrm{m}^{2} \mathrm{~s}^{-1}$, with the range in $\kappa_{H}$ being less a reflection of site-to-site variability, and more due to uncertainties in the background strain rate acting on the patch combined with uncertain time dependence. The Site I (low strain) experiment exhibited minimal stretching, elongating to approximately $10 \mathrm{~km}$ over 6 days while maintaining a width of $\sim 5 \mathrm{~km}$, and with a notable vertical tilt in the meridional direction. By contrast, the Site II (intermediate strain) experiment exhibited significant stretching, elongating to more than $50 \mathrm{~km}$ in length and advecting more than $150 \mathrm{~km}$ while still maintaining a width of order $3-5 \mathrm{~km}$. Early surveys from both experiments showed patchy distributions indicative of small-scale stirring at scales of order a few hundred meters. Later surveys show relatively smooth, coherent distributions with only occasional patchiness, suggestive of a diffusive rather than stirring process at the scales of the now larger patches. Together the two experiments provide important clues as to the rates and underlying processes driving diapycnal and isopycnal mixing at these scales.
\end{abstract}

\section{Introduction}

Lateral dispersion is among the fundamental physical processes, along with advection and diapycnal mixing, that determine the distribution and fate of water masses, nutrients, and other dissolved and particulate tracers in the ocean. Lateral dispersion occurs on scales ranging from centimeters to hundreds of kilometers, and can be driven by a host of mixing and stirring processes, with no

\footnotetext{
${ }^{\text {a }}$ Current affiliation: Mitsubishi Electric Research Laboratories, Cambridge, Massachusetts.

${ }^{\mathrm{b}}$ Deceased.
}

Corresponding author: Miles A. Sundermeyer, msundermeyer@ umassd.edu one process governing all scales (e.g., Richardson 1926; Okubo 1971). Certain scales of mixing and stirring, particularly lateral scales of order $0.1-30 \mathrm{~km}$ (termed here the submesoscale, as they fall between 3D microscale turbulence and large-scale stirring by mesoscale eddies), are notoriously difficult to measure. As a result, processes driving dispersion at these scales are still poorly understood. State-of-the-art numerical models resolve some of the relevant processes. However, processes occurring at or near the grid scale $(0.1-1 \mathrm{~km}$ for regional models and $10-30 \mathrm{~km}$ for global synoptic and/or climate models) must be parameterized.

The importance of submesoscale dispersion in the ocean is widespread. A growing body of literature shows that submesoscale processes play a key role in transferring energy between the mesoscale and the smallest 
scales that ultimately lead to dissipation (e.g., Thomas et al. 2008; Molemaker et al. 2010; D'Asaro et al. 2011; McWilliams 2016, and references therein). While mesoscale stirring inarguably dominates lateral spreading of water properties on basin scales, the fate of fronts and filaments generated by the forward cascade of tracer variance to small scales also depends strongly on the submesoscale (Capet et al. 2008a,b; Thomas et al. 2008; Molemaker et al. 2010; Mahadevan et al. 2010; Lévy et al. 2012). There is also growing evidence that vertical velocities associated with certain submesoscale (and often ageostrophic) processes play an important role in fluxing mass, buoyancy, potential vorticity (PV), and tracers between the mixed layer and the pycnocline (e.g., Mahadevan and Tandon 2006; Thomas et al. 2008), including $\mathrm{CO}_{2}$, nutrients, and other biologically important dissolved constituents (Mahadevan and Archer 2000; Martin 2003; Mahadevan et al. 2011; Lévy et al. 2012). In short, the classic separation between mesoscale dynamics dominating the large scale and the microscale dominating the small scale has become increasingly blurred, with the submesoscale mutually affecting, as well as communicating between, the two limits.

Numerous submesoscale processes also lead to enhanced dispersion in and around fronts in the ocean (e.g., Mahadevan 2006; Thomas et al. 2008). Among them are mesoscale strain and classic frontogenesis driven by ageostrophic motions (Hoskins and Bretherton 1972), both of which can increase small-scale tracer variance and enhance large-scale stirring. There is also a suite of instabilities acting within or below the surface mixed layer (e.g., Thomas et al. 2008; Fox-Kemper et al. 2008; Molemaker et al. 2005; Taylor and Ferrari 2010). Some of these are fed by energy from the mesoscale, others by wind forcing, and still others require negative buoyancy forcing. Under certain conditions, multiple mechanisms may be at play simultaneously (Mahadevan and Tandon 2006). Diagnosing which mechanisms are active in a specific situation can be challenging even in the best of circumstances, in part because measurements of the underlying processes are often limited in both time and space.

To address these challenges, a major field campaign was organized by the Office of Naval Research under a Department Research Initiative (DRI) entitled "Scalable Lateral Mixing and Coherent Turbulence," nicknamed LatMix. The primary objective of LatMix was to provide a better understanding, through observations, modeling, and theoretical studies, of the physical processes driving lateral dispersion, and to translate this understanding into parameterizations and formulae that are applicable under a variety of forcing conditions. Beyond mechanism-specific investigations, questions to be addressed included better understanding of how mesoscale and submesoscale mixing processes are linked via the transfer of energy, tracer variance and/or PV; whether relevant submesoscale mixing processes are inherently both vertical and horizontal; and whether such processes can lead to efficient mixing across ocean fronts, which are otherwise thought to be barriers to transport. Within LatMix, a 2-yr field program was developed to investigate which processes can explain observed isopycnal dispersion rates. Field sites were selected by identifying key features of the mechanisms of interest, and then determining in a general sense what regions of the ocean would provide the strongest signal and highest probability of exhibiting those phenomena. To provide a maximum range of conditions, the first field effort, conducted in 2011, targeted typical open ocean conditions within a mesoscale eddy field of moderate eddy kinetic energy, away from persistent fronts, bathymetric influence, and other strong forcing. By contrast, the second field effort, conducted in 2012, focused on strong frontal conditions, including strong wind and buoyancy forcing and high available potential energy.

In this paper, we present the results from two rhodamine dye and drogued-drifter experiments conducted during the first LatMix field deployment. This first deployment took place from 26 May to 20 June 2011, and was a coordinated effort between three research vessels and a Navy P3 aircraft, which was on site periodically during the experiment. More than 20 principal investigators participated from approximately a dozen institutions around the United States, bringing a variety of in situ, remote sensing, theoretical, and numerical modeling efforts to bear. A broad overview of the LatMix 2011 field experiment is provided by Shcherbina et al. (2015). The dye and drifter studies were supported jointly by the LatMix DRI and grants from the National Science Foundation.

The organization of this paper is as follows. In section 2, we describe the overall field program, along with the site characteristics for the two rhodamine dye releases. We also describe the methods of dye injection, surveying, data processing, and analysis. Sections 3 and 4 describe the evolution of the dye patches and the resulting inferences regarding diapycnal and isopycnal dispersion. Section 5 compares the two experiments, highlighting similarities and differences between them, and pointing to potential signatures of underlying processes. Finally, section 6 summarizes and concludes.

\section{Methods}

\section{a. Overview of field program}

The LatMix 2011 field experiment was conducted in the Sargasso Sea, approximately $250 \mathrm{~km}$ southeast of 

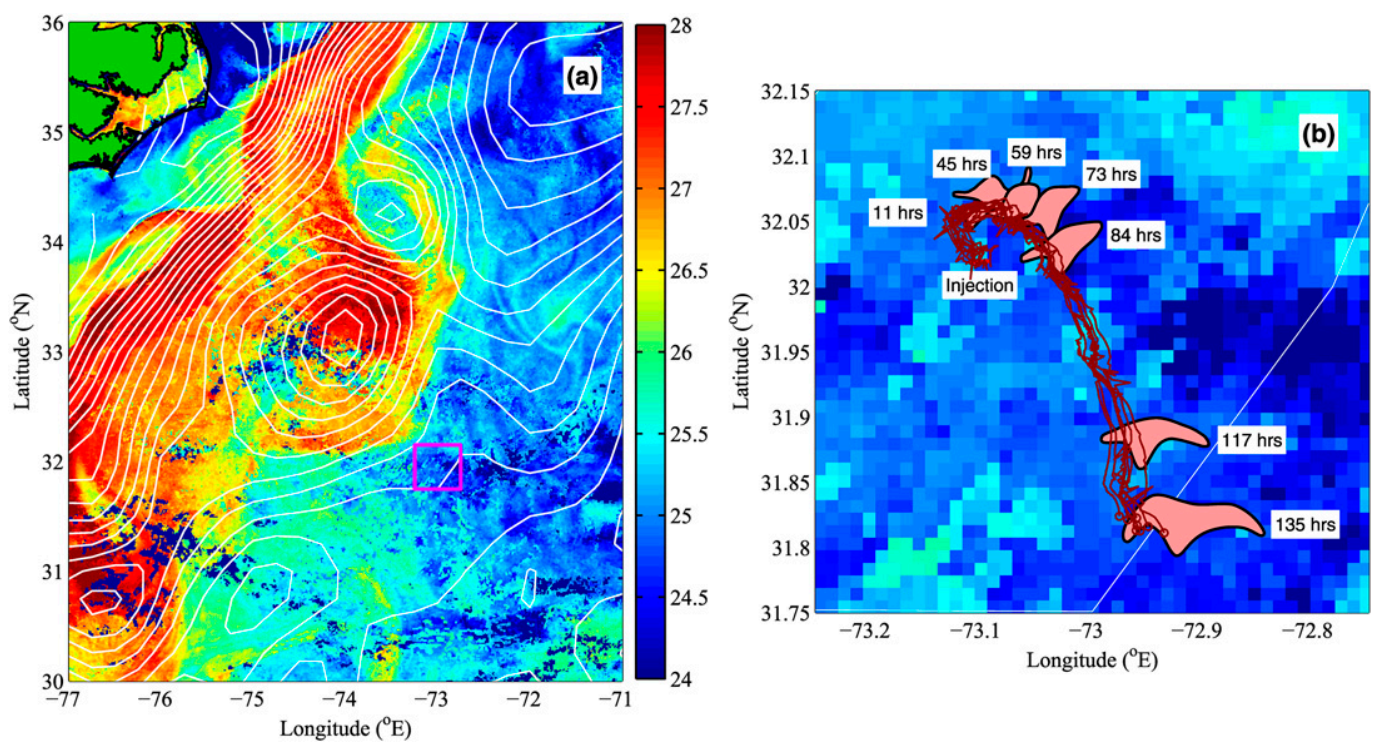

FIG. 1. (a) Location of the LatMix 2011 low strain rate study site showing sea surface temperature $\left({ }^{\circ} \mathrm{C}\right)$ on 4 Jun 2011, the day of the first dye injection, with AVISO satellite-derived sea surface height contours overlaid in white. The location of the Site I dye experiment is shown as a magenta box in (a). (b) The evolution of the drogued drifters (red lines) and approximate distribution of the dye patch during successive surveys (light red patches with corresponding times since injection).

Cape Hatteras, North Carolina, far enough east and south of the Gulf Stream to be considered "typical" open ocean conditions (Fig. 1). Three ships and a Navy P3 Orion aircraft conducted coordinated in situ and remote sampling of the water column, fluorescent dye tracers, meteorological, and optical properties of the area. Specific roles of each ship were as follows. Dye and drogued drifters (J. Ledwell and M. Sundermeyer's groups from WHOI and University of Massachusetts Dartmouth, respectively; and M. Levine's group from Oregon State University), and Lagrangian floats (E. D'Asaro's group at University of Washington) were deployed and primarily sampled from R/V Cape Hatteras. Electromagnetic-Autonomous Profiling Explorer (EM-APEX) floats were deployed and tended by R/V Endeavor (T. Sanford's group at University Washington), along with four gliders (R. K. Shearmann's group from Oregon State University), in concert with 10 -km scale hydrographic surveys in and around the dye patches throughout the experiments (J. Klymak's group, University of Victoria, British Columbia). Last, R/V Oceanus conducted $30-\mathrm{km}$ scale hydrographic surveys using a Triaxus towed instrument package (C. Lee's group at University of Washington), and tended both a T-REMUS autonomous underwater vehicle (L. Goodman, formerly at University of Massachusetts Dartmouth), and a Hammerhead conductivity-temperature-depth (CTD) and temperature microstructure sensor package (E. Kunze's group, formerly at University of Victoria, British Columbia).
Experiments were conducted in late May/early June, sufficiently late in spring for warming to have established a seasonal pycnocline at approximately 30 40-m depth, capped by a surface mixed layer ranging from 10- to 20-m depth; but not so far into summer to encounter significant tropical storm or hurricane activity. Additionally, the field location featured optical properties that were favorable for the airborne lidar component of the experiments (diffuse attenuation $K_{d} \sim 0.03 \mathrm{~m}^{-1}$ ), while also being within reasonable flight distance for airborne surveys of the dye patches. While the airborne lidar surveys were a significant and integral part of the LatMix 2011 field experiments, discussion of their results will be presented in a separate manuscript (M. A. Sundermeyer et al. 2020, unpublished manuscript).

Within the general field location, two sites were selected for the two main 6-day rhodamine dye release experiments. Site I was chosen following an initial broadscale survey of the region as the "low strain" site (horizontal strain rate $\sim 10^{-6} \mathrm{~s}^{-1}$, or $\sim 0.01 f$ ), outside of any mesoscale eddies or enhanced strain rate between eddies (see Fig. 1). Site II was similarly chosen after a second broadscale survey, this time as a region of "moderate strain" (horizontal strain rate $\sim 10^{-5} \mathrm{~s}^{-1}$, or $\sim 0.1 f$ ) near a saddle point of flow between multiple mesoscale eddies (see Fig. 10, to be discussed in the next section). Wind speeds for both experiments were weak to moderate, ranging from 


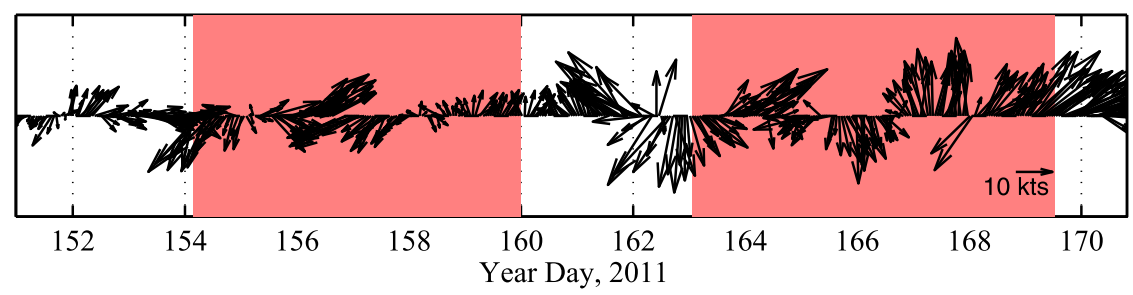

FIG. 2. Wind as measured from R/V Cape Hatteras during the LatMix 2011 field experiment. Red shaded regions indicate periods from injection to the end of the final survey of the two rhodamine dye experiments.

0 to $15 \mathrm{kt}\left(1 \mathrm{kt} \approx 0.51 \mathrm{~m} \mathrm{~s}^{-1}\right)$ (Fig. 2 ; see also Shcherbina et al. 2015).

\section{b. Dye and drifters}

Upon selection of suitable locations, two fluorescent dye releases were conducted within the seasonal pycnocline using rhodamine WT. In studies such as these, rhodamine WT has many favorable qualities-it is inert, is nontoxic at the concentrations typically used, does not readily adsorb onto particles within the water column, and it does not readily photo-decay in sunlight (e.g., Smart and Laidlaw 1977; Wilson et al. 1984). It can also be measured in situ using a towed fluorometer to concentrations as low as $0.01 \mathrm{ppb}$.

Dye injections were performed using a custom built injection system consisting of a Sea-Bird 9plus CTD sensor mounted to a stainless steel frame. The frame was made neutrally buoyant by attaching floats, and tethered to a large depressor weight $(\sim 100 \mathrm{~kg})$, which in turn was attached to the ship's hydrographic wire. The purpose of the tethered arrangement was to help isolate the CTD from the heaving motion of the ship during injection, while at the same time keeping the injection system at a controlled depth via the weight. The injection frame was equipped with a polyvinyl chloride (PVC) diffuser attached to a hose, which in turn was attached to the CTD cable via cable ties as it was lowered over the side of the ship. CTD data were fed back to the laboratory to enable real-time monitoring and winch control. This arrangement allowed the dye to be pumped onto a nominal target density surface at depth, with the operator having the ability to make minor adjustments to the depth of the injection system as the depth of the target isopycnal varied during the injection.

Prior to each release, CTD casts were made to determine a background density profile, and to identify a suitable injection density and depth. The density of the dye solution was adjusted on deck to within $1 \mathrm{~kg} \mathrm{~m}^{-3}$ of the target density by mixing it with isopropyl alcohol and freshwater, while also accounting for the thermal expansion coefficient of the mixture and its temperature relative to the target density surface. The ballasted dye was continuously pumped to depth at a rate of approximately $0.2 \mathrm{~L} \mathrm{~s}^{-1}$, such that each injection lasted approximately $1 \mathrm{~h}$. We estimate a $\sim 100$-fold dilution of the dye solution with ambient seawater in the immediate turbulent wake of the injection sled, bringing the injection solution to within $0.001-0.01 \mathrm{~kg} \mathrm{~m}^{-3}$ of the target density. While turbulent dilution in the wake of the injection sled is critical in helping erase the injection mixture density anomaly, it also creates an initial diapycnal spread of the dye. An additional purpose of moving during the release is to ensure that the dye experiences a variety of mixing events from the start, and thus is not dominated by a single mixing event.

Along with the dye, drogued drifters were released during the injections both as an aid to tracking the dye, and to obtain independent estimates of strain rate and lateral dispersion. The drifters employed Comtech Trackpacks affixed to a $30-\mathrm{cm}$ spar atop a $41-\mathrm{cm}$ mooring ball, all tethered via $0.3-\mathrm{cm}$ Maxibraid to a 6.3-m holey sock drogue. Text messages containing GPS locations were sent by each drifter at hourly intervals via the GlobalStar network, and relayed from shore back to the ship, enabling near-real-time drifter tracking. Prior to each injection, drogue tether lengths were set so that the center of each drogue was at approximately the target dye injection depth. For each release, nine drogued drifters were deployed in a cross pattern centered on the dye streak; the first five deployed during injection, the last four immediately following injection in a perpendicular track approximately midway along the injection line. Additional drifters were also deployed during secondary fluorescein experiments in the near vicinity of the rhodamine dye patch, such that as many as 18 drogued drifters were in the water at any given time. Results from these additional dye/drifter studies will be reported in a forthcoming publication.

Following injection, the dye was immediately surveyed first using a Moving Vessel Profiler (MVP, first survey only), and then by a towed SeaSciences Acrobat tow sled (all subsequent surveys). Both systems were equipped with a combination of CTD and dye fluorometers-an 
AML Micro CTD and Seapoint fluorometers for the MVP, and an RBR CTD and Turner Cyclops-7 fluorometers for the Acrobat. The advantage of the free-fall MVP sampling system was that it enabled higher resolution $(\sim 50 \mathrm{~m})$ along-track sampling early after release when the dye patches were still small. Complementing this, for later surveys (i.e., after the first day), the Acrobat sampling system provided more rapid, but still relatively high resolution sampling $(\sim 100-150 \mathrm{~m})$ once the patches had grown to many kilometers in length and width.

For both dye experiments, primary objectives of the sampling surveys were to (i) delimit the dye patch horizontally and vertically, and (ii) provide multiple transects through the patch to enable characterization of both diapycnal and isopycnal moments of the tracer distribution. To this end a sampling strategy was adopted in which, prior to each survey, an initial estimate of the dye patch location was obtained based on either the injection location, or on prior sampling of the patch. Prior known locations of the dye were advected to the current time using real-time shipboard acoustic Doppler current profiler (ADCP) velocity measurements at the dye injection depth and assuming that the velocity field was uniform in space but varied in time. This information was combined with real-time locations of the drogued drifters released in and around the dye to provide a running best estimate of the likely location and size of the dye patch. Within a given survey, realtime advection of the ship track helped avoid repeated sampling of the same water parcels, while for subsequent surveys it helped with planning where to next look for the patch.

\section{c. Data reduction and processing}

The MVP used during the initial surveys of the two experiments executed tethered free-fall profiles. Sampling at $25 \mathrm{~Hz}$, its CTD and fluorometers provided a vertical profile resolution of $\sim 3-4 \mathrm{~cm}$. MVP data were corrected for thermister response time (to address salinity spiking) by applying a time lag to the temperature measurements relative to conductivity. Since the MVP fluorometer was mounted within the semi-enclosed casing of the instrument package, it experienced an additional delay due to the flushing time of its sensing volume. To correct for this, an inverse exponential filter was applied to the MVP fluorometer data, with neighboring up-/downcasts used to optimize the time constant for the inverse exponential. Despite these corrections, the MVP data showed a consistent bias in its salinity. Assuming a relatively uniform vertical stratification spanning the dye patch, which was the case for the present experiments, this bias likely had the greatest effect on the first moment of dye in $z$, as this moment relies most directly on the precise value of density. Since the second moment in $z$ is controlled by the spread of dye about its vertical center of mass, it was less impacted by this bias. Additionally, the same bias would not have significantly impacted our estimates of lateral moments, since these are driven by lateral distributions of dye on isopycnals rather than the specific density value of those isopycnals. As noted in sections 3 and 4, with these considerations in mind, we do not use the initial MVP surveys when we compute the first moments of the dye patch in $z$, but do use them for estimating the remaining moments.

The Acrobat CTD/fluorometer system used in the remaining surveys executed a tow-yo sawtooth pattern. Sampling at $6 \mathrm{~Hz}$, its CTD and fluorometers provided a vertical profile resolution of $\sim 10 \mathrm{~cm}$ over the central portion of its profiling range, with finer resolution near the top and bottom of the casts. The Acrobat data were corrected for thermister response time, again by applying a time lag to the temperature measurements relative to conductivity. Additionally, an inverse exponential filter was applied to the Acrobat fluorometer data to account for instrument sensor lag. Finally, to maximize the recovery of very low dye concentrations (down to $\sim 0.01 \mathrm{ppb}$ ), all Acrobat fluorometer data were processed to remove a low frequency $(\sim 0.004 \mathrm{~Hz})$ sinusoidal modulation in their noise floor, which was found in both the rhodamine and fluorescein raw time series. The source of this modulation was presumably electronic, as it was very regular, yet uncorrelated with any other aspect of the Acrobat and CTD sampling. The noise floor modulations were removed by fitting a running sinusoid to the baseline dye signal after removing much shorter lived peaks generated as the tow-yo package passed vertically through the dye patch.

After applying the corrections described above, all data were separated into equivalent up- and downcasts. For both sampling platforms each cast was binned in isopycnal coordinates, and then referenced back to a "stretched" pressure coordinate using the mean density-pressure relationship averaged over the entire experiment. This procedure removed heaving of isopycnals by internal waves and other pressuredensity variations occurring on the scale of the dye patch, making the vertical and horizontal diffusivities computed here effectively diapycnal and isopycnal diffusivities, respectively.

Diapycnal diffusivities were estimated for each experiment as the time rate of change of the vertical second moment of the dye distribution averaged over each survey, 


$$
\kappa_{z}=\frac{1}{2} \frac{\partial \sigma_{z}^{2}}{\partial t}
$$

where

$$
\sigma_{z}^{2}=\frac{\int_{-\infty}^{\infty} z^{2} C d z}{\int_{-\infty}^{\infty} C d z}-\left(\frac{\int_{-\infty}^{\infty} z C d z}{\int_{-\infty}^{\infty} C d z}\right)^{2}
$$

is the second moment of dye concentration $C$ in $z$. The first term on the rhs of (2) represents the spread of the patch across isopycnals, while the second is the square of the vertical center of mass (i.e., the square of the first moment in $z$ ). The procedure for computing vertical first and second moments was as follows. For each survey we first selected all dye profiles that sampled the entire patch thickness, that is, those profiles whose dye signal exceeded the detection limit somewhere within the profile, and which also fell below the detection limit at the upper and lower extremes of the cast. This screening minimized bias in vertical second moment calculations due to incomplete dye profiles. All qualified profiles were then averaged in density space, and the result related back to "stretched" pressure using the mean density-pressure relationship for the entire experiment. The second moment $\sigma_{z}^{2}$ relative to the vertical center of mass of the dye was then computed for each survey per (2), and the time rate of change of $\sigma_{z}^{2}$ computed via linear regression to obtain $\kappa_{z}$ per (1). Notable here is that the first moment of the dye in $z$, that is, the vertical center of mass, did not remain fixed throughout the experiments, but rather became shallower (toward lighter density) relative to the injection depth over the course of both experiments. As will be explained in sections 3 and 4, the reason for this is believed to be solar warming of the dye layer. This notwithstanding, the effects of the movement of the center of mass on the second moment are still accounted for in (2).

Isopycnal diffusivities were computed taking into account the lateral strain rate, which tended to elongate the patch in one horizontal direction relative to the other. Following Sundermeyer and Ledwell (2001), we assume a simple horizontal advection/diffusion model of the form

$$
\frac{\partial \theta}{\partial t}+\gamma x \frac{\partial \theta}{\partial x}-\gamma y \frac{\partial \theta}{\partial y}=\kappa_{H} \nabla_{H}^{2} \theta,
$$

where $\theta$ represents dye concentration on an isopycnal surface, $\gamma=\partial u / \partial x=-\partial v / \partial y$ is a horizontally nondivergent strain rate, and is $\kappa_{H}$ an isotropic isopycnal diffusivity. Here a coordinate system is assumed that aligns with the principle axis of the strain tensor, with $x$ and $y$ corresponding to the directions of the major and minor axes of the patch, respectively. In contrast to Sundermeyer and Ledwell (2001), for the present analysis we assume that both $\gamma$ and $\kappa_{H}$ are constant in space and time within a given portion of the experiment (i.e., 2-3 days; we consider full time dependence of the observations in a follow-on paper by D. A. Birch et al. (2020, unpublished manuscript). Multiplying (3) by $x^{2}$ and integrating over $x$, or by $y^{2}$ and integrating over $y$, we obtain expressions for how the second moments of tracer in $x$ and $y$ vary in time. Approximating the initial condition as a Gaussian, that is,

$$
\theta=\theta_{0} \exp \left(-\frac{x^{2}}{2 \sigma_{x}^{2}}-\frac{y^{2}}{2 \sigma_{y}^{2}}\right)
$$

the second moments of tracer in the $x$ and $y$ directions are given by

$$
\frac{d \sigma_{x}^{2}}{d t}-2 \gamma \sigma_{x}^{2}=2 \kappa_{H}
$$

and

$$
\frac{d \sigma_{y}^{2}}{d t}+2 \gamma \sigma_{y}^{2}=2 \kappa_{H}
$$

Notable in (5) and (6) are the different signs of the straining terms. In the direction of the major axis of the patch (i.e., along-streak, here the $x$ direction), straining causes $\sigma_{x}^{2}$ to increase exponentially, while diffusion increases $\sigma_{x}^{2}$ linearly - thus for steady strain, the growth of streak length always becomes exponential after sufficient time. Meanwhile, in the direction of the minor axis (i.e., cross-streak, here the $y$ direction), straining leads to a convergence of the tracer patch, opposing the tendency of diffusion to widen the streak (or at least limit its infinitesimal thinning). This stretching and narrowing is shown schematically in Fig. 3a. Key to estimating diffusivity is that (5) and (6) represent two equations for two unknowns, $\gamma$ and $\kappa_{H}$, that is, given variances $\sigma_{x}$ and $\sigma_{y}$ associated with the major and minor axes of the tracer patch, plus their time rate of change, it is possible to solve uniquely for both unknowns.

\section{d. Estimating along-/cross-streak moments}

To determine isopycnal diffusivity and horizontal strain rate from the dye distributions we first rotate the observations into a reference frame aligned with the major/minor axes of the tracer patch. Given our assumption of horizontally uniform strain rate, we further account for curvature of the tracer streak, effectively 
(a)

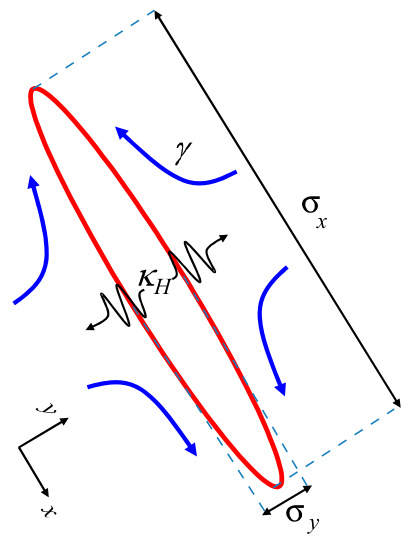

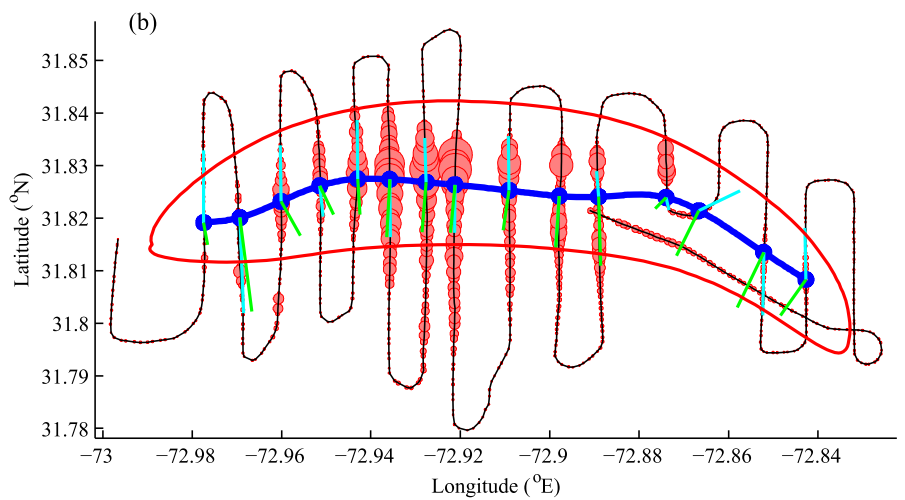

FIG. 3. (a) Schematic of strain-diffusion model used to infer second moments $\sigma_{x}$ and $\sigma_{y}$; along- and across-streak strain rate $\gamma$ and effective lateral diffusivity $\kappa_{H}$ of observed dye patches. (b) Example in plan-view of observed dye distribution during the Site I experiment showing the ship track (black curve) advected relative to the dye center of mass, with red circles indicating vertically integrated dye concentration measured along each tow-yo cast. The red curved ellipse indicates the approximate envelope of the dye patch, with the bold blue line indicating the estimated major axis of the dye streak. Light blue "flags" indicate the direction of the ship track relative to the local major axis, and light green "flags" indicate local normal direction relative to the major axis.

unfolding the tracer patch by considering local alongand cross-streak directions along the major axis of the patch. An example of this for the first dye experiment is shown in Fig. 3b. For each survey, the approximate orientation of the patch is typically already determined in the field, as it is easiest to sample the dye transverse to the major axis of strain rate and then work along the major axis in successive transects through the patch. For the first dye experiment, the along-streak direction was approximately east-west, such that cross-streak transects were approximately north-south. For the second experiment, the major axis was northwest-southeast, so that cross-streak transects were southwest-northeast.

From each cross-streak transect, we next compute the center of mass and second moment of dye as a function of isopycnal using an expression similar to (2), but in the along-track direction. To obtain the effective diffusivity strictly along isopycnals, excluding the effects of vertical shearing, we first compute horizontal moments on isopycnals, and then average these moments with depth. This is in contrast to first vertically integrating the dye, and then computing horizontal moments, since the latter would incorporate vertical shearing into the overall horizontal moment (see, e.g., Sundermeyer and Ledwell 2001). In computing depth-averaged moments, depth dependent isopycnal moments are additionally weighted by the mean vertical dye concentration profile for each transect before depth averaging. This yields a single depth-averaged center of mass and horizontal second moment for each transect that represents the depth-averaged advection and spreading of the dye patch, independent of effects of vertical/isopycnal shear.
An additional measure of interest, which is being used explicitly in ongoing analysis (D. A. Birch et al. 2020, unpublished manuscript) is the depth averaged vertical shear experienced by the patch, revealed by the tilt in the horizontal center of mass of the patch across the transect (e.g., see Figs. 6 and 13), to be discussed in the next sections.

Having computed along-transect centers of mass and second moments, we identify the major axis of the dye streak during each survey as the curve connecting each transect's depth-averaged center of mass (bold blue curve in Fig. 3b). This procedure assumes that a major and minor axis (i.e., along- versus across-streak) direction can be clearly defined, which in the two experiments presented here was indeed the case. We next identify the angle at which each transect crossed the major axis of the streak, and project each transect onto the local normal to the streak axis before computing its alongtrack second moment. Having identified a single depth averaged cross-streak isopycnal second moment for each transect, we average these values, weighted by the integrated dye concentration for each transect to obtain a single cross-streak horizontal second moment for each survey.

Finally, to compute along-streak moments of the dye patch for each survey, we reconstruct an equivalent along-streak transect along the major axis of the dye patch using the cross-streak-integrated dye concentration profiles for each transect. From this reconstructed transect, we again compute the center of mass and second moment as a function of isopycnal, and the weighted depth averaged second moment in the along-streak 
direction. Similar to the cross-streak direction, we also compute the tilt of the vertical center of mass of dye in the along-streak direction, again as a measure of the time-integrated vertical shear acting in the along-streak direction of the patch.

\section{e. Estimating isopycnal dispersion and strain rate}

Having computed along- and cross-streak second moments for each of the approximately daily surveys of the two dye experiments, we compute the effective isopycnal diffusivity $\kappa_{H}$ via one of two approaches. First, for the first experiment only (see explanation in following sections) we perform a least squares regression to the cross-streak second moments of tracer as a function of time, assuming the growth of the streak width is due strictly to diffusion, with zero strain rate $\gamma$. Second, we compute $\kappa_{H}$ and $\gamma$ jointly by making the additional simplifying assumption that after a time of order $1 / \gamma$, the strain rate dominates diffusion in the along-streak direction, while the competing effects of the strain rate and diffusion yield a steady streak width in the acrossstreak direction. In this latter case, (5) and (6) simplify to

$$
\frac{d \sigma_{x}^{2}}{d t}-2 \gamma \sigma_{x}^{2}=0,
$$

and

$$
\gamma \sigma_{y}^{2}=\kappa_{H} .
$$

While this assumption may be tenuous for the first dye experiment, as will be discussed below, it is certainly reasonable for the second. In practice, (7) enables $\gamma$ to be determined directly from the growth rate of the along-streak second moment; the familiar exponential stretching. Having obtained $\gamma, \kappa_{H}$ can be estimated from (8) based on both $\gamma$ and the estimated cross-streak second moment $\sigma_{y}^{2}$.

\section{Site I: Low strain region}

\section{a. Site I description}

As noted above, the location for the first dye release was chosen as a region of low horizontal strain rate, of order $10^{-6} \mathrm{~s}^{-1}(0.01 \mathrm{f})$, outside of any mesoscale eddies, and also outside of any enhanced strain rate between eddies. Specifically, satellite remote sensing images of sea surface temperature (e.g., NOAA CoastWatch/OceanWatch) showed sea surface temperature variability, but no indication of coherent warm- or cold-core eddies or rings (see Fig. 1). Similarly, sea surface height maps derived from satellite altimeter (e.g., www.aviso.oceanobs.com/duacs/) revealed no mesoscale eddies at the site (see also Fig. 1). Upon arriving at the prospective site, ship-based hydrographic ADCP surveys (not shown; see Shcherbina et al. 2015) further confirmed weak velocity and strain rate fields, and corresponding weak lateral density gradients.

Pre-injection hydrographic surveys as well as those conducted over the course of the dye experiment showed a surface mixed layer extending to $\sim 15 \mathrm{~m}$, with a seasonal pycnocline below (Fig. 4). Buoyancy frequencies below the mixed layer ranged from 0.013 to $0.023 \mathrm{rad} \mathrm{s}^{-1}$, decreasing gradually with depth. Weather was generally fair throughout the experiment, with winds ranging from 2 to $10 \mathrm{~m} \mathrm{~s}^{-1}$, either out of the west/southwest or northeast (e.g., see Fig. 2). Insolation was also typical of fair summertime conditions, yielding peak daytime heat flux into the ocean of $\sim 800 \mathrm{~W} \mathrm{~m}^{-2}$. The latter is significant in that this insolation plus the optical clarity of the water apparently led to net warming of both dye patches over the course of the two 6-day experiments.

\section{b. Site I injection and dye surveys}

The Site I rhodamine injection was performed on 4 June 2011 at approximately 0335 UTC at $32.015^{\circ} \mathrm{N}$, $73.10^{\circ} \mathrm{W}$, at a nominal depth of $30.33 \pm 1.38 \mathrm{~m}$, and a target density of $\sigma_{\theta}=25.422 \pm 0.014 \mathrm{~kg} \mathrm{~m}^{-3}$, where error bars are given as plus or minus one standard deviation of values measured by the CTD during the injection. The injection lasted a total of $1.1 \mathrm{~h}$, which at a ship speed of $0.5-1 \mathrm{kt}$ resulted in an initial dye streak approximately $1.4 \mathrm{~km}$ in length. A total of $98 \mathrm{~kg}$ of rhodamine dye was injected. Allowing for the collapse of the turbulent wake behind the injection sled (e.g., Lin and Pao 1979), and assuming a resulting width of the tracer streak after a few hours of $\sim 100 \mathrm{~m}$, we estimate an initial dye concentration of order $120 \mathrm{ppb}$.

Nine drogued drifters were released during the first dye injection, five approximately evenly spaced along the injection line, and four more bisecting the injection line to form an approximate "+" sign. A single Lagrangian float equipped with a CTD, dye fluorometer, and acoustic Doppler velocimeter was also released midway through the injection. The float measured detailed small-scale structure (order $1 \mathrm{~m}$ ) of the dye, as well as vertical shear across the float (E. D'Asaro 2011, personal communication). The latter is used in ongoing analysis to help evaluate the possible importance of vertical shear dispersion (D. A. Birch et al. 2020, unpublished manuscript).

Dye surveying commenced immediately following injection, beginning with the OSU MVP (day 1) and then using the UMass Acrobat (days 2-6). During this 


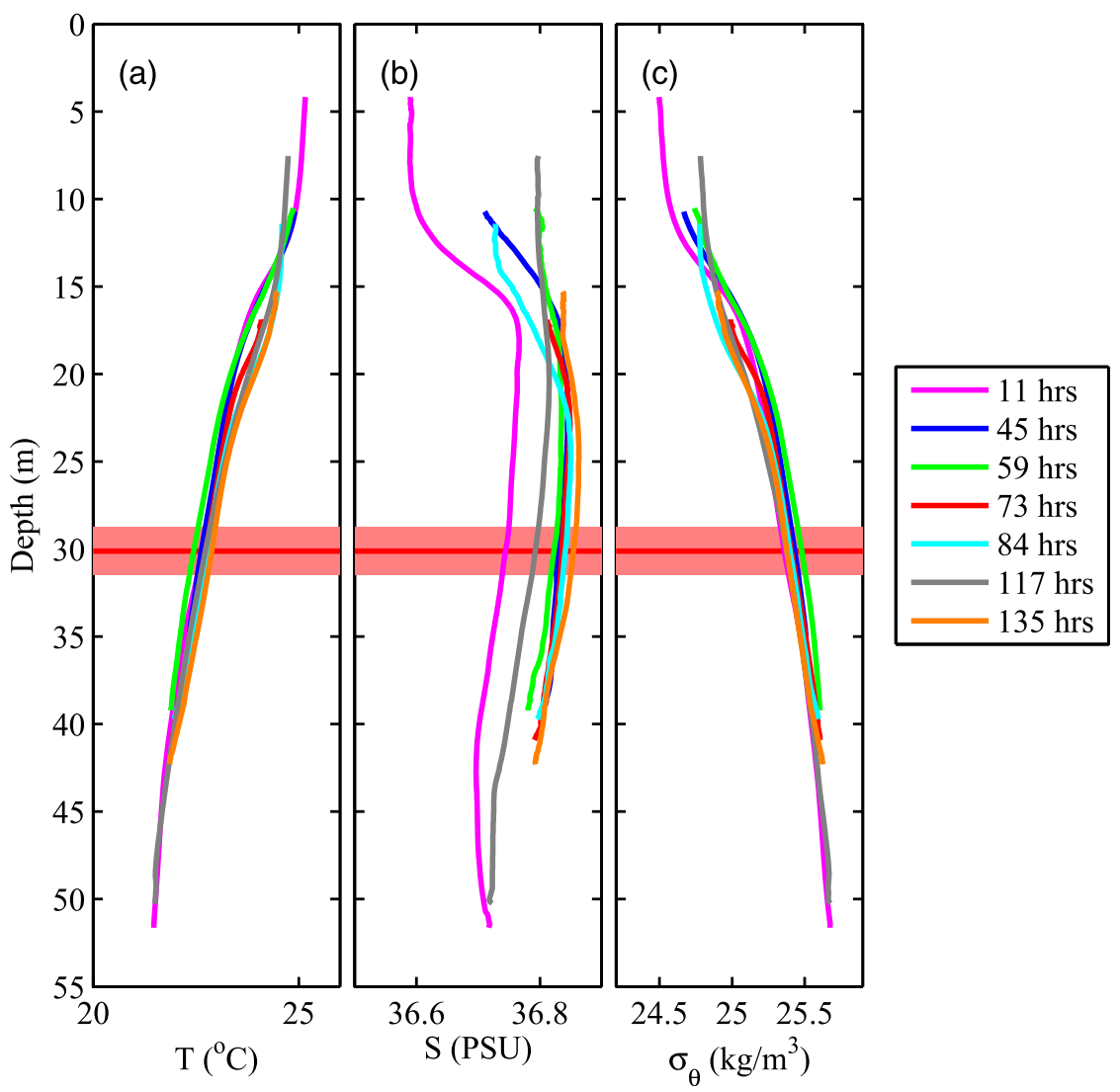

FIG. 4. Mean (a) potential temperature, (b) salinity, and (c) potential density profiles as a function depth for seven dye surveys conducted during the Site I rhodamine dye experiment showing a $\sim 15-\mathrm{m}$ mixed layer overlying a seasonal pycnocline and gradually decaying stratification with depth. The legend shows elapsed time since the end of dye release to the middle of each survey. The red line with light red shading indicates the target injection depth plus or minus one standard deviation.

period, the patch drifted southeast approximately $30 \mathrm{~km}$, growing from its initial injection scale of $\sim 100 \mathrm{~m} \times 1.5 \mathrm{~km}$ to approximately $5 \mathrm{~km}$ wide by $10 \mathrm{~km}$ long. Seven surveys delimiting the dye patch were performed. Plan view maps of the dye during these surveys are shown in Fig. 5. An example of a transect through the dye patch during the final survey of the first experiment is shown in Fig. 6.

\section{c. Site I diapycnal diffusivity}

Following the approach described in section 2, mean dye concentration profiles were computed for each survey as a function of potential density, and then converted to a vertical depth coordinate using the mean density-depth relation averaged over the experiment. Vertical dye distributions for the Site I experiment are shown in Fig. 7. Considering both the vertical center of mass and vertical second moment as a function of time, we found a consistent trend in both parameters, with the center of mass moving to lighter water over the 6-day experiment at an equivalent vertical velocity of
$(6.2 \pm 1.0) \times 10^{-6} \mathrm{~m} \mathrm{~s}^{-1}$, and the second moment growing approximately linearly in time corresponding to a diapycnal diffusivity of $(2.3 \pm 1.9) \times 10^{-6} \mathrm{~m}^{2} \mathrm{~s}^{-1}$, where uncertainties are based on $95 \%$ confidence intervals computed via ordinary least squares regression (Fig. 8). Note that in estimating these parameters, we have included the mean injection density in our regression fit to the first moment in $z$, but have excluded it in the fit to the second moment in $z$ (i.e., Fig. $8 \mathrm{a}$ vs Fig. 8b). This is because the first moment, which was more or less measured directly during the injection as the mean injection density, is better known than the second moment, which we presume was additionally modified by the turbulent wake of the injection sled. Meanwhile, for reasons described in section $2 \mathrm{c}$, due to a salinity bias in the data from the first (MVP) dye survey, this survey was used in estimating growth in the second moment in $z$, but not the first moment in $z$.

Regarding the apparent movement of the center of mass toward lighter water, it is notable that the mean 


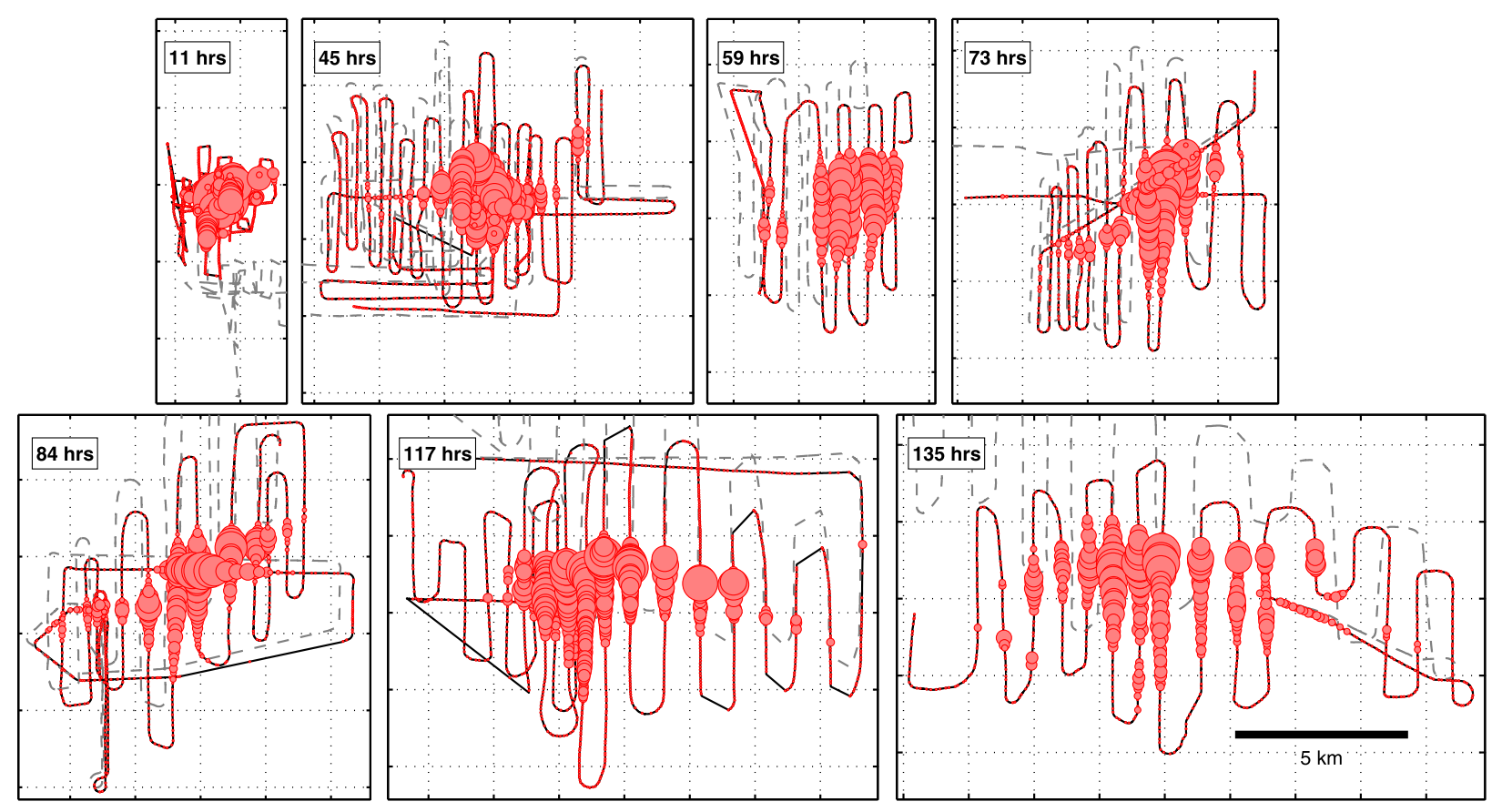

FIG. 5. Successive ship-based dye surveys conducted during the Site I rhodamine dye experiment. Elapsed time since end of dye release to the middle of survey is indicated in each subplot. Gray dashed and black solid lines indicate, respectively, ship track in unadvected Earth coordinates and advected using shipboard ADCP data. Red circles indicate vertically integrated dye concentration, with size of circle indicating relative amount of dye during each survey. Maximum circle size varies by survey. All surveys are plotted on the same scale following the approximate center of mass of the evolving patch.

temperature of the dye patch increased over the course of the experiment, but the salinity did not (not shown). The clarity of the water as well as the fair weather and significant insolation during the experiment make it likely that the observed increase in temperature and hence decrease in density of the dye patch was caused by solar warming. Here we assume that heating by insolation is

$$
\frac{d T}{d t}=-\frac{1}{c_{p} \rho} \frac{d I}{d z}
$$

where $T$ is temperature, $t$ time, $c_{p}$ the specific heat capacity of seawater, $\rho$ the density of seawater, and $I$ the solar irradiance; and further that irradiance decays exponentially with depth as

$$
I=I_{0} \exp \left[-\int_{z}^{0}\left(\alpha_{\text {sw }}+\alpha_{\text {dye }}\right) d z\right],
$$

where $\alpha_{\text {sw }}$ and $\alpha_{\text {dye }}$ are seawater and dye attenuation/ absorption coefficients. The average short wave solar radiation incident at the surface during the experiments was approximately $200 \mathrm{~W} \mathrm{~m}^{-2}$. Downwelling radiation as a function of depth and wavelength in the background seawater was measured with a profiling spectrometer (Satlantic ACS). The absorption coefficient of Rhodamine-WT dye as a function of wavelength had been measured in the laboratory with a spectrofluorometer, and the quantum efficiency of emission is approximately 0.6 . With these data, plus the observed dye distribution as a function of depth and time (averaged over the lateral extent of the dye patch), we constructed a simplified radiative transfer model. This model indicated that radiative heating would result in an upward displacement of the dye relative to the injection density surface of approximately $3.5 \mathrm{~m}$ over the 6 -day period of the experiment, in agreement with the observations. The model also indicated that radiative heating decreases enough with depth to amplify the growth of the second moment with time across isopycnals, and thus to favor the lower part of the range of diapycnal diffusivity given above.

\section{d. Site I isopycnal diffusivity and strain rate}

Horizontal second moments of the dye patch for each survey were computed following the approach described in section 2c. For the first experiment, the dye patch elongated primarily in the east-west direction, obtaining a length approximately 2.5 times 


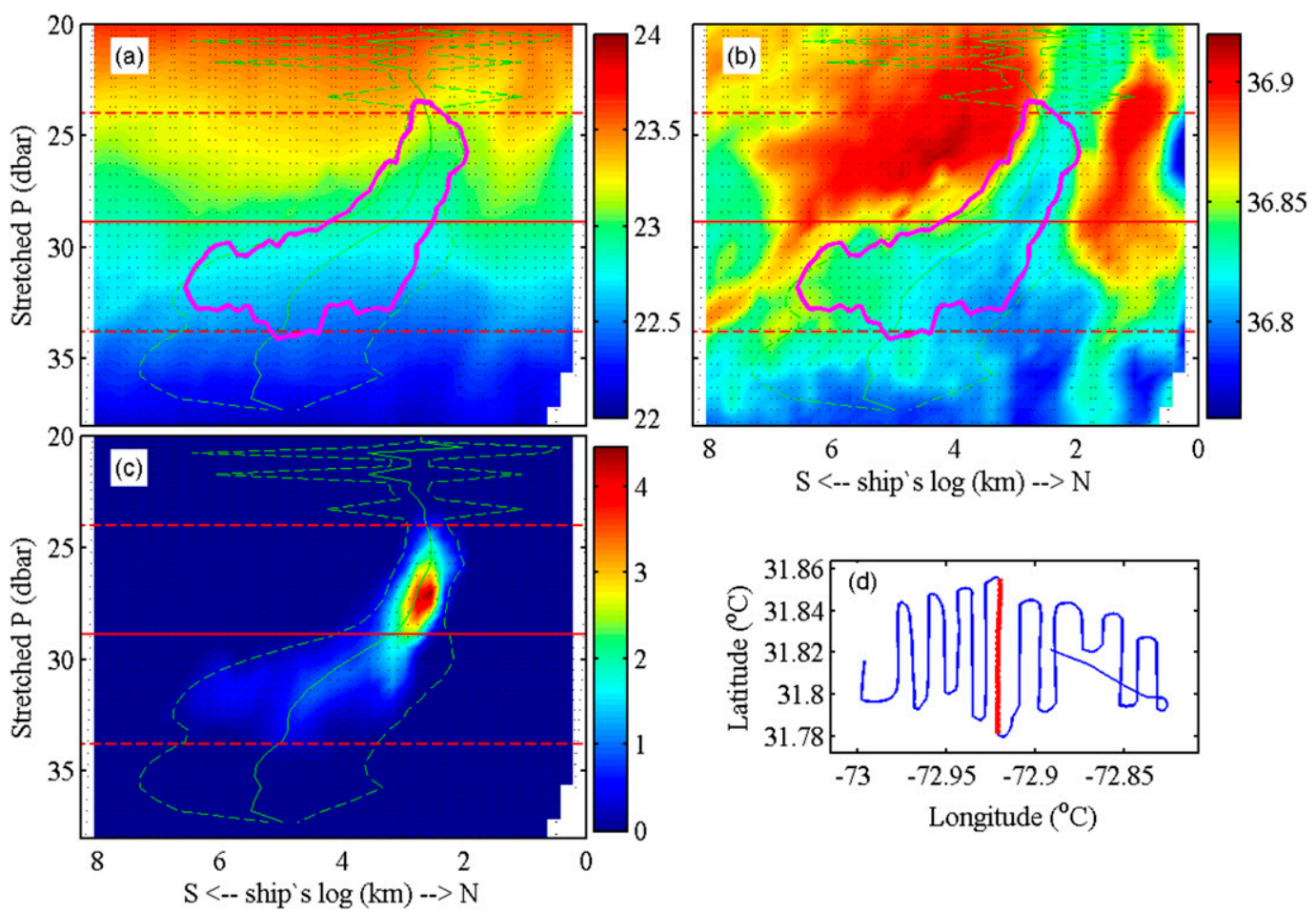

FIG. 6. Example of (a) temperature, (b) salinity, and (c) dye concentration measured during a single transect during the final survey of the first rhodamine dye experiment, and (d) plan view of the location of the transect (red line) relative to the full survey (blue line). In (a)-(c), $T, S$, and dye concentration are plotted with ship's log in the $x$ axis and potential density, converted back to pressure via the mean density pressure relationship for the survey. Red horizontal lines in (a)-(c) indicate the vertical center of mass (solid) \pm 2 standard deviations (dashed). Green vertically varying lines indicate horizontal center of mass (solid) \pm 2 standard deviations as measured along the transect. Magenta curves in (a) and (b) indicate for reference the dye concentration contour containing $95 \%$ of the tracer measured during the transect.

its width after 6 days (Fig. 5). Using our estimates of the injection length combined with the time evolution of the major axis of the patch, we estimate a mean strain rate per (7) of $\gamma=(3.0 \pm 0.6) \times 10^{-6} \mathrm{~s}^{-1}$ (Fig. 9a). This value is roughly consistent with independent estimates from drogued drifters, which ranged from $(0.1-2) \times 10^{-5} \mathrm{~s}^{-1}$ over the course of the experiment.

Considering the time evolution of cross-streak second moment of the dye patch (Fig. 9b), the width of the patch did not follow the steady state assumption represented by (8). Rather, the second moment in the cross-streak direction grew throughout the 6-day experiment. Our expectation is that the strain rate should balance diffusion after of order $1 / \gamma=1 / 3.0 \times 10^{-6} \mathrm{~s}^{-1} \approx$ 4 days. Retreating from the steady state assumption, if we ignore the effects of the strain rate and simply assume that diffusivity is given by the time rate of change of the second moment [i.e., setting $\gamma=0$ in (5)], we obtain $\kappa_{H}=0.4 \pm 0.2 \mathrm{~m}^{2} \mathrm{~s}^{-1}$. Alternatively, pressing on with (8) and using our inferred value of strain rate together with a typical cross-streak variance during the early part of the experiment (e.g., days 2-4 surveys in Fig. 9b), we obtain a slightly larger estimate, $\kappa_{H}=0.7 \pm 0.1 \mathrm{~m}^{2} \mathrm{~s}^{-1}$. Finally, using an upper estimate of strain rate inferred from the drogued drifters, $\gamma=1 \times 10^{-5} \mathrm{~s}^{-1}$, and the cross-streak variance typically observed during the later surveys (e.g., 4.5-6-day surveys in Fig. 9b), we obtain $\kappa_{H}=2.8 \pm$ $1.4 \mathrm{~m}^{2} \mathrm{~s}^{-1}$. Which of these values is most correct depends on what assumptions one is willing to accept-our view here is that none of our underlying assumptions are perfect, but that our estimates provide lower and upper bounds of the true value. A more refined estimate of $\kappa_{H}$ that takes into account temporal variability of the observed growth in the dye moments, and also considers the integrated effects of vertical shear on the tilt of the patch, is being conducted as part of ongoing analysis (D. A. Birch et al. 2020, unpublished manuscript). Findings thus far are roughly in the middle of the present range of estimates, that is, also of order $\kappa_{H} \sim O(1) \mathrm{m}^{2} \mathrm{~s}^{-1}$. 

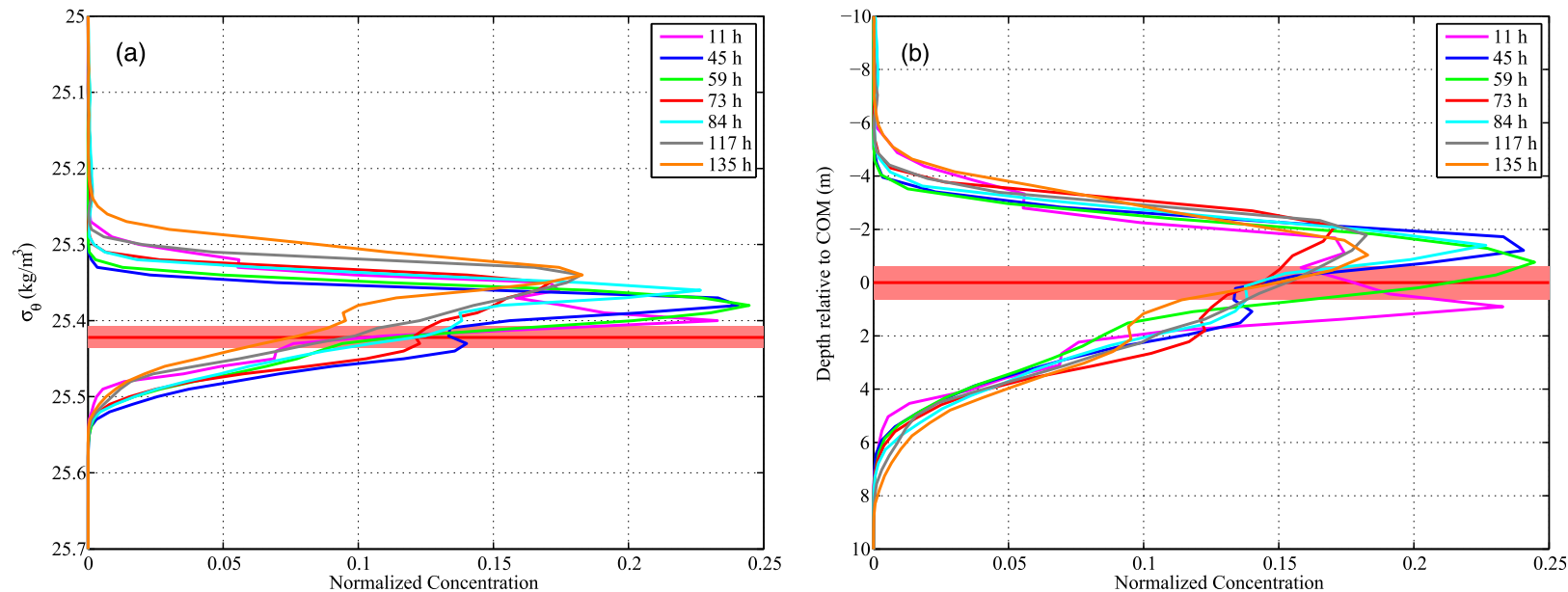

FIG. 7. Normalized dye concentration profiles during the first rhodamine dye experiment as a function of (a) potential density and (b) depth relative to the vertical center of mass. Colors represent different surveys. Legend gives time in hours from dye release to the midpoint of each survey. The red line with light red shading indicates the target injection depth plus or minus one standard deviation.

\section{Site II: Intermediate strain region}

\section{a. Site II description}

The location for the second dye release was chosen as a region of intermediate strain rate, of order $10^{-5} \mathrm{~s}^{-1}$ $(0.1 f)$, in the near proximity of a saddle point between multiple mesoscale eddies. Specifically, satellite remote sensing combined with ship-based surveys showed a confluence region with corresponding front formation and diffluence aligned approximately northwest-southeast. A map of satellite derived sea surface temperature, corresponding sea surface height, and the location of the dye injection are shown in Fig. 10. Again, preinjection hydrographic surveys revealed a rich subsurface structure in and around the study region, with a surface mixed layer extending to approximately $15-20 \mathrm{~m}$, and a seasonal pycnocline below (Fig. 11). Buoyancy frequencies below the mixed layer ranged from 0.014 to $0.020 \mathrm{rad} \mathrm{s}^{-1}$, decreasing gradually with depth. In addition to the vertical density structure, there was also a $T / S$ front in the mixed layer and opposing front just below it (not shown; see Kunze et al. 2015).
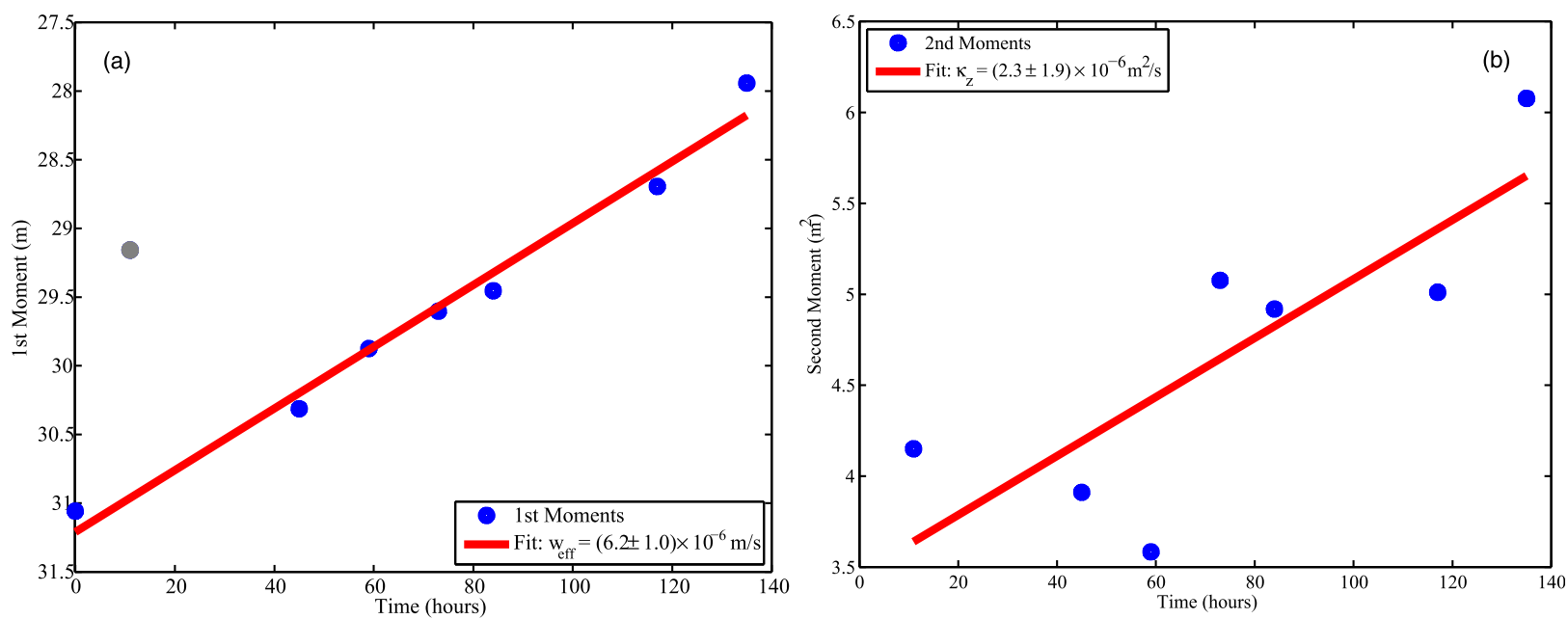

FIG. 8. Time evolution of (a) center of mass and (b) second moment in $z$ of dye distribution for the first rhodamine dye experiment. Bold red lines are linear fits to the data, with $95 \%$ confidence intervals of inferred vertical velocity $w_{\text {eff }}$ and diapycnal diffusivity $\kappa_{z}$ given in the legends. The gray data point in (a) is from the first survey conducted using the OSU MVP, which was not used in the regression for $w_{\text {eff }}$, but was used in (b) for the regression for $\kappa_{z}$ (see text for explanation). Conversely, moments for the dye injection streak $(t=0)$ were used in (a), but not in (b) (again, see text for explanation). 

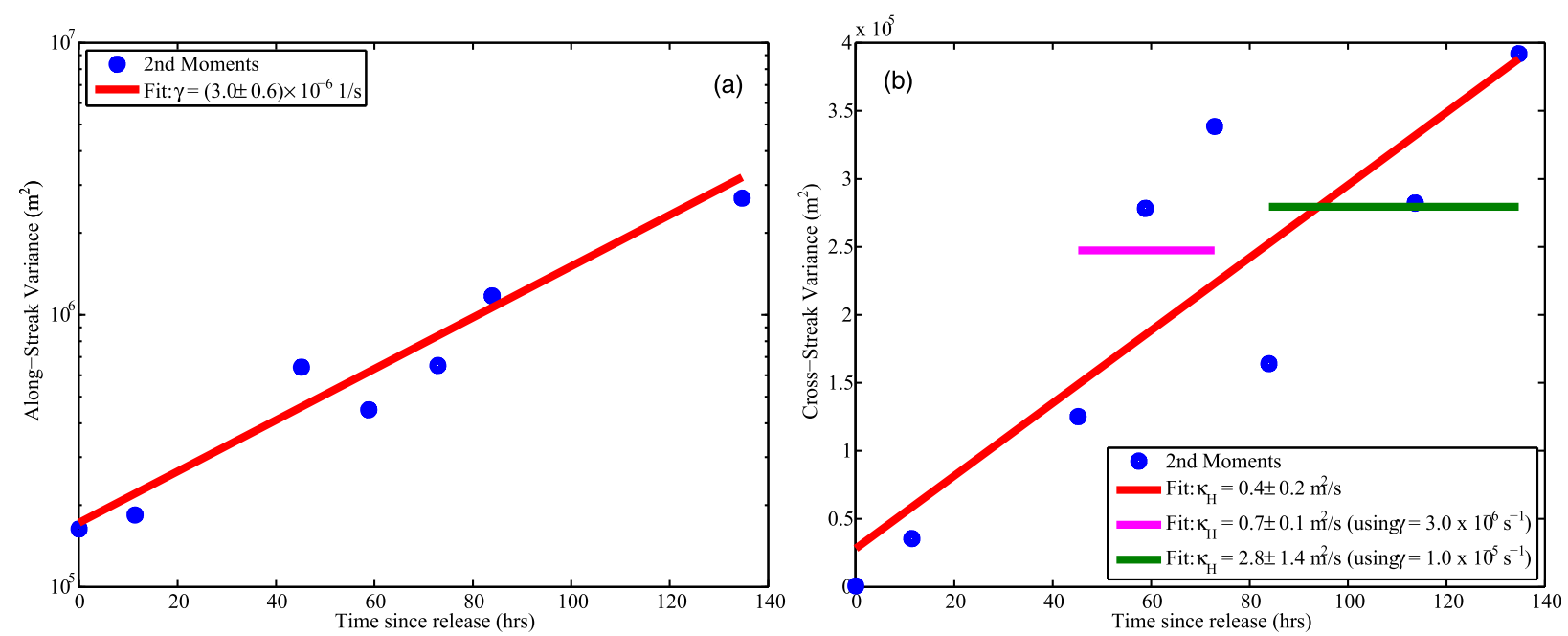

FIG. 9. Time evolution of the second moment in (a) the along-streak, and (b) the cross-streak direction for the first rhodamine dye experiment. Bold red lines are linear fits to the data, with $95 \%$ confidence intervals of inferred horizontal strain rate $\gamma$ and isopycnal diffusivity $\kappa_{H}$ given in the legends. Additional estimates of $\kappa_{H}$ inferred from a balance between convergent strain rate and cross-streak isopycnal diffusivity per (8) are indicated in the legend of (b) and described further in the text.

The weather was again generally fair throughout the second experiment, with the notable exception of two cold fronts that passed over the study site on days 1 and 5 following the injection, both of which brought periods of thunderstorms, wind, and rain. With the exception of the first of these two storm events, winds were again modest, ranging from 2 to $10 \mathrm{~m} \mathrm{~s}^{-1}$ out of varying directions during the first half of the experiment, then persistently from the south/southwest during the second half of the experiment (see Fig. 2). Insolation remained typical of fair summertime conditions, again with the exception of the first of the two storm events
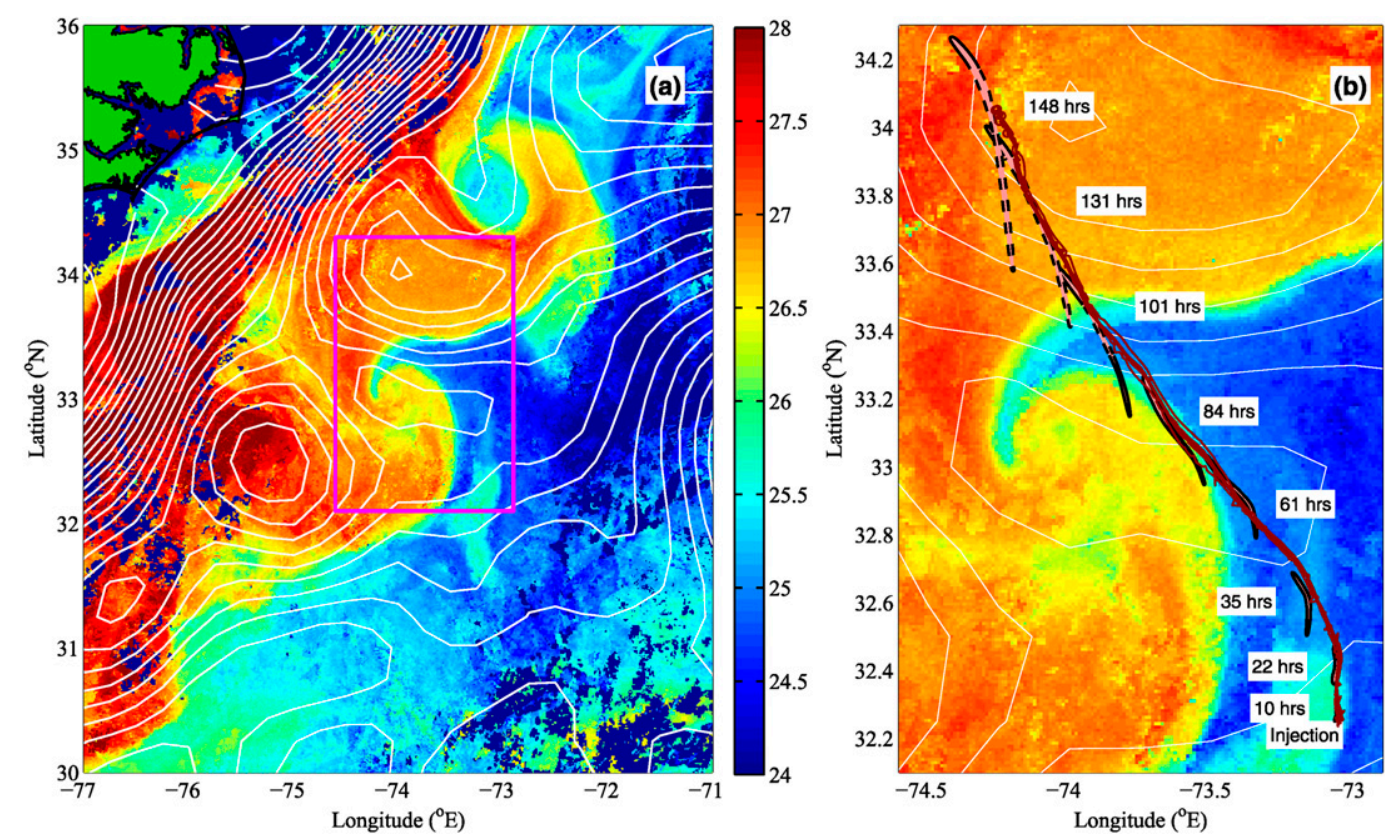

FIG. 10. (a) Location of the LatMix 2011 intermediate strain rate study site showing sea surface temperature $\left({ }^{\circ} \mathrm{C}\right)$ on 13 Jun 2011, the day of the second dye injection, with AVISO satellite-derived sea surface height contours overlaid in white. The location of the Site II dye experiment is shown as a magenta box in (a). (b) The evolution of the drogued drifters (red lines) and approximate distribution of the dye patch during successive surveys (light red patches with corresponding times since injection). 

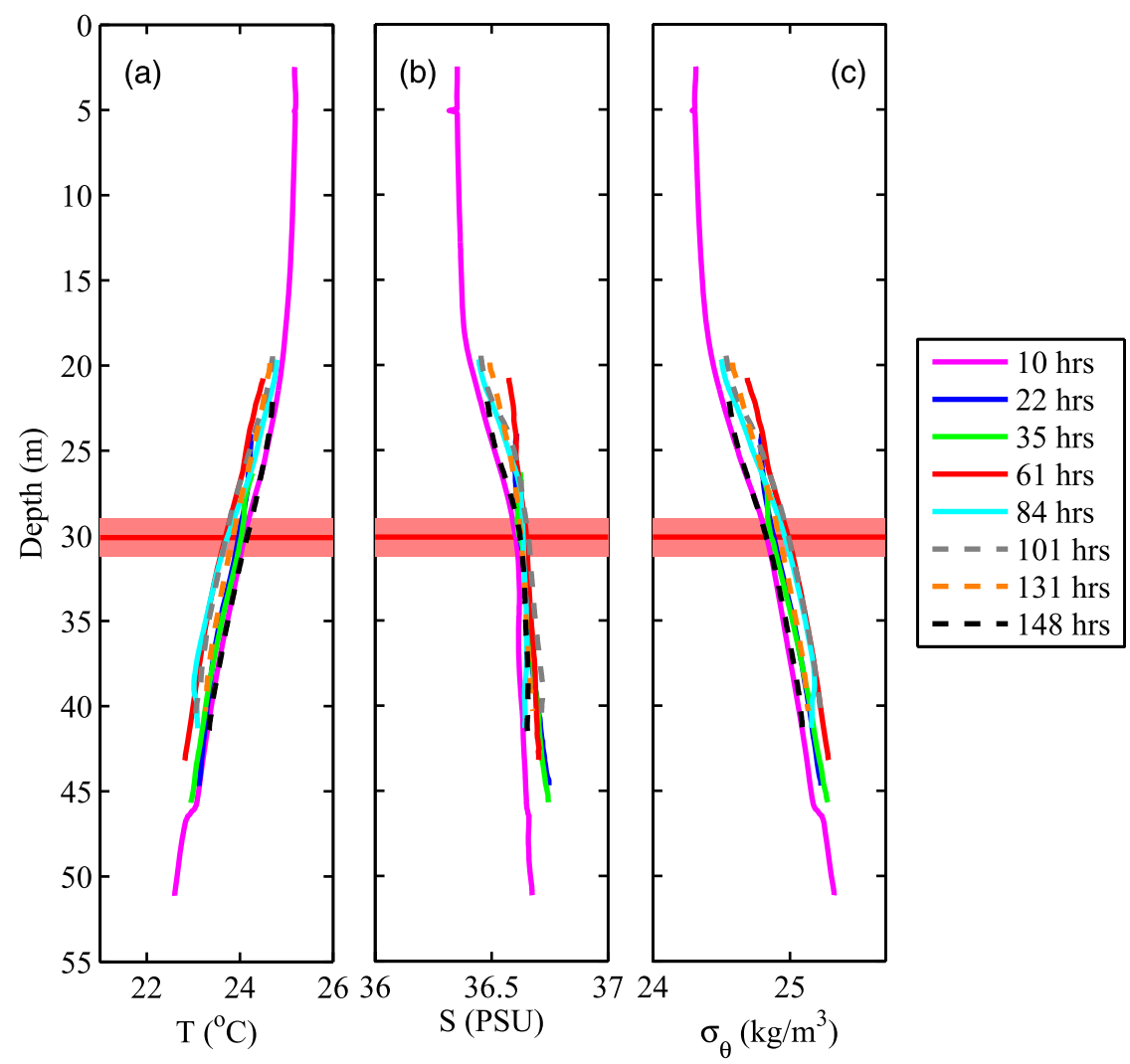

FIG. 11. Mean (a) potential temperature, (b) salinity, and (c) potential density profiles as a function depth for seven dye surveys conducted during the Site II rhodamine dye experiment showing a $\sim 15-20-\mathrm{m}$ mixed layer overlying a seasonal pycnocline and gradually decaying stratification with depth, similar to Fig. 4.

around 1 day after injection (the second set of thunderstorms occurred during night time hours).

\section{b. Site II injection and dye surveys}

The Site II rhodamine injection was performed on 13 June 2011 at approximately 0100 UTC at $32.24^{\circ} \mathrm{N}$, $73.055^{\circ} \mathrm{W}$, at a nominal depth of $30.32 \pm 1.14 \mathrm{~m}$, and a target density of $\sigma_{\theta}=25.045 \pm 0.012 \mathrm{~kg} \mathrm{~m}^{-3}$, where again error estimates are given as \pm 1 standard deviation. Similar to the first experiment, the injection lasted a total of $1.1 \mathrm{~h}$, yielding an injection streak length of approximately $1.8 \mathrm{~km}$, and an initial dye concentration of order $100 \mathrm{ppb}$. A total of $105 \mathrm{~kg}$ of rhodamine dye was injected during the second release. Again nine GPStracked drogued drifters were released during the dye injection, five spaced along the injection line, and four bisecting it to make an approximate "+" sign, and with a single Lagrangian float released midway through the injection.

Surveying of the dye patch again commenced immediately following injection, first using the OSU MVP, then the UMass Acrobat. Over the next 6 days, the dye proceeded to advect northwestward, translating its center of mass more than $150 \mathrm{~km}$, and stretching to more than $50 \mathrm{~km}$ long, all the while maintaining a streak width of between 3 and $5 \mathrm{~km}$. Eight surveys were performed over 6 days, six of which delimited the patch, and two of which captured only its northmost reaches (Fig. 12).

As in the first experiment, transect views of the dye showed marked $T-S$ structure accompanying the patch, with the dye lying along a local maximum in both temperature and salinity extending over its entire depth range (Fig. 13). Over the eight dye surveys, the dye patch closely followed this $T-S$ structure as it evolved. As in the first experiment, the patch remained mostly coherent throughout the experiment, maintaining a single connected streak that stretched lengthwise, showing little evidence of significant meanders except at the northernmost extent of the patch toward the end of the experiment.

\section{c. Site II diffusivity and strain rate}

Repeating the analysis used for the first experiment, mean dye concentration profiles were computed for 

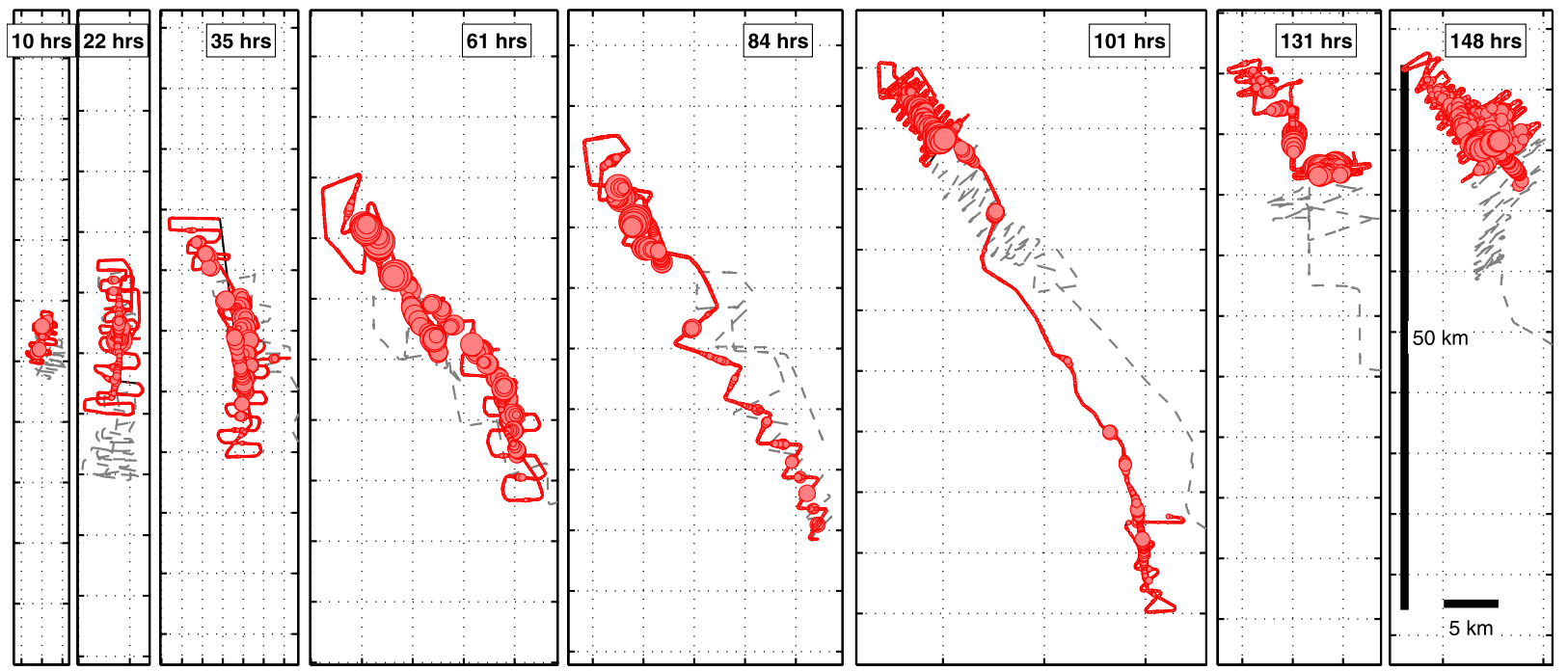

FIG. 12. Successive ship-based dye surveys conducted during the Site II rhodamine dye experiment, similar to Fig. 5.

each survey following the methodology described in section 2c (Fig. 14). Different from the first experiment, however, in estimating vertical and horizontal moments here we were mindful that the final two surveys of the second experiment did not sample the entire patch and hence may present a biased view. Again, both the vertical center of mass and vertical second moment showed consistent trends, with the vertical center of mass yielding an equivalent vertical velocity of $(5.2 \pm 2.2) \times 10^{-6} \mathrm{~m} \mathrm{~s}^{-1}$, and the second moment yielding a diapycnal diffusivity of $(4.8 \pm 4.5) \times$ $10^{-6} \mathrm{~m}^{2} \mathrm{~s}^{-1}$ based on the first four surveys alone, or

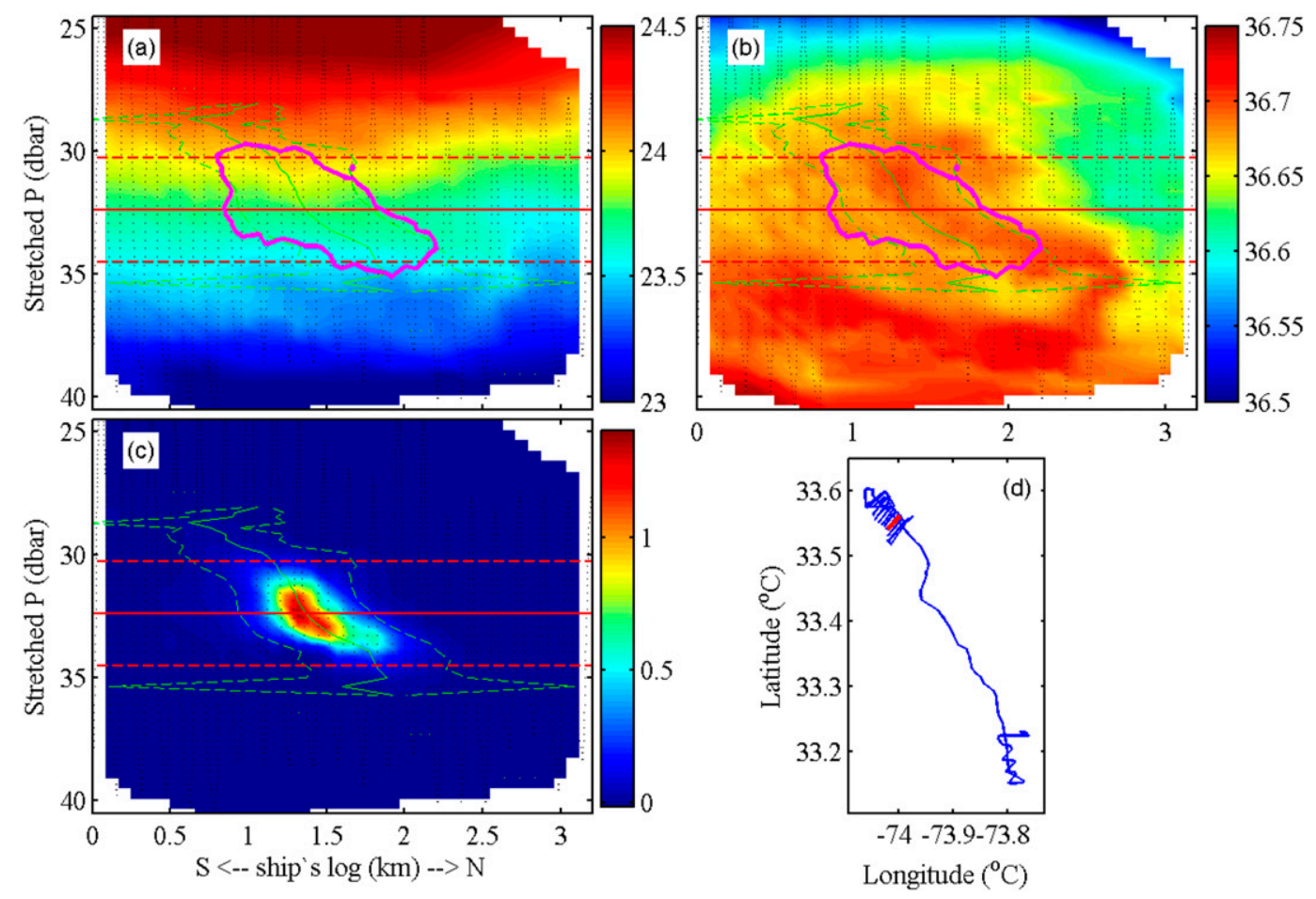

FIG. 13. Example of (a) temperature, (b) salinity, and (c) dye concentration measured during a single transect during the sixth survey of the second rhodamine dye experiment, and (d) plan view of the location of the transect (red line) relative to the full survey (blue line), similar to Fig. 6. 

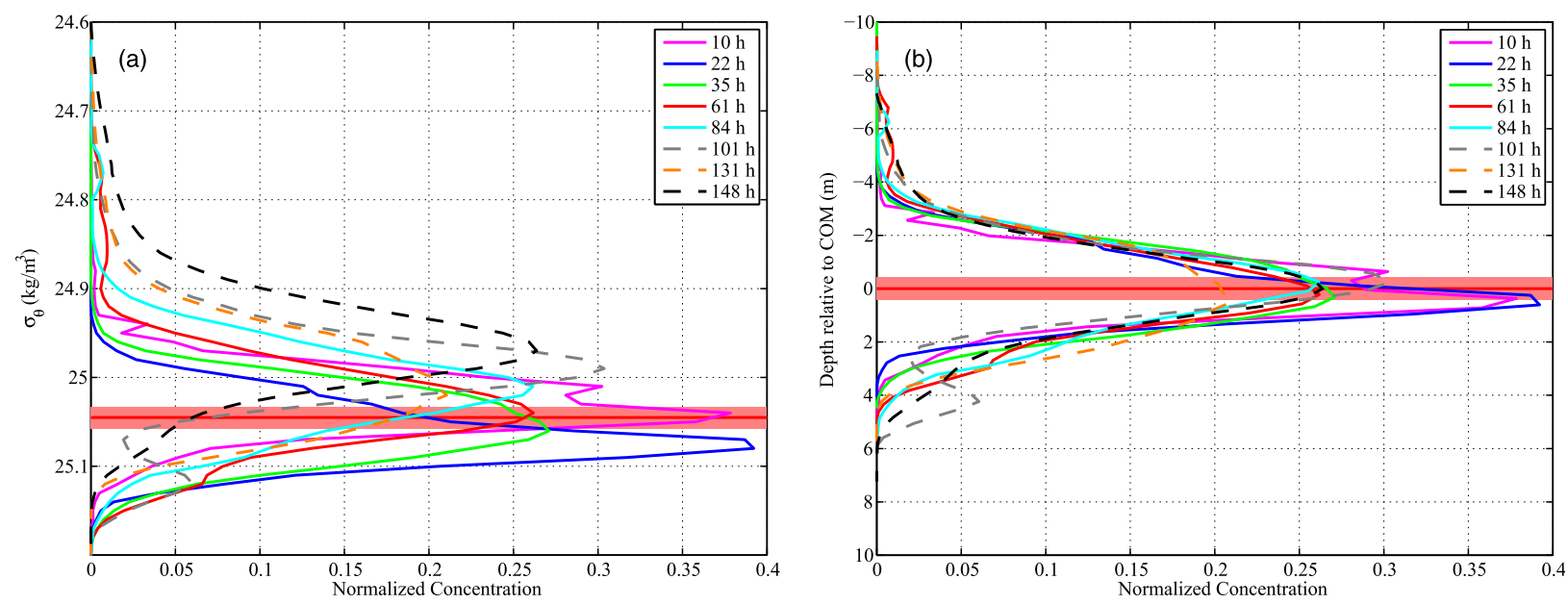

FIG. 14. Normalized dye concentration profiles during the second rhodamine dye experiment as a function of (a) potential density and (b) depth relative to the vertical center of mass, similar to Fig. 7.

$(2.3 \pm 1.1) \times 10^{-6} \mathrm{~m}^{2} \mathrm{~s}^{-1}$ based on all the surveys (Fig. 15). Again, the observed warming of the patch was roughly consistent with that expected from solar heating.

Horizontal second moments of the dye patch were again computed using the methodology described in section 2. As noted above, in the second dye experiment the patch advected northwestward more than $150 \mathrm{~km}$ over 6 days, stretching to $50 \mathrm{~km}$ by day 4 , and presumably longer in the two days thereafter when only incomplete surveys of the patch were obtained (Figs. 12 and 16a). Using (7) to estimate the rate of strain acting on the patch, we find $\gamma=(1.7 \pm 0.4) \times 10^{-5} \mathrm{~s}^{-1}$ early in the evolution of the patch (days 0-2), decreasing to $(2.5 \pm 3.6) \times 10^{-6} \mathrm{~s}^{-1}$ later in the evolution (days 2-4). Again, these values (including the trend from higher to lower strain rates) are roughly consistent with those inferred from drifter separation rates, which varied from $(3-5) \times 10^{-5} \mathrm{~s}^{-1}$ early versus later in the evolution.

The time evolution of the cross-streak second moment again showed little evidence of a steady state (Fig. 16b), which is not surprising given the sixfold variation in the strain rate experienced by the patch over the course of the experiment. Again assuming that the streak width adjusts to the strain rate on a
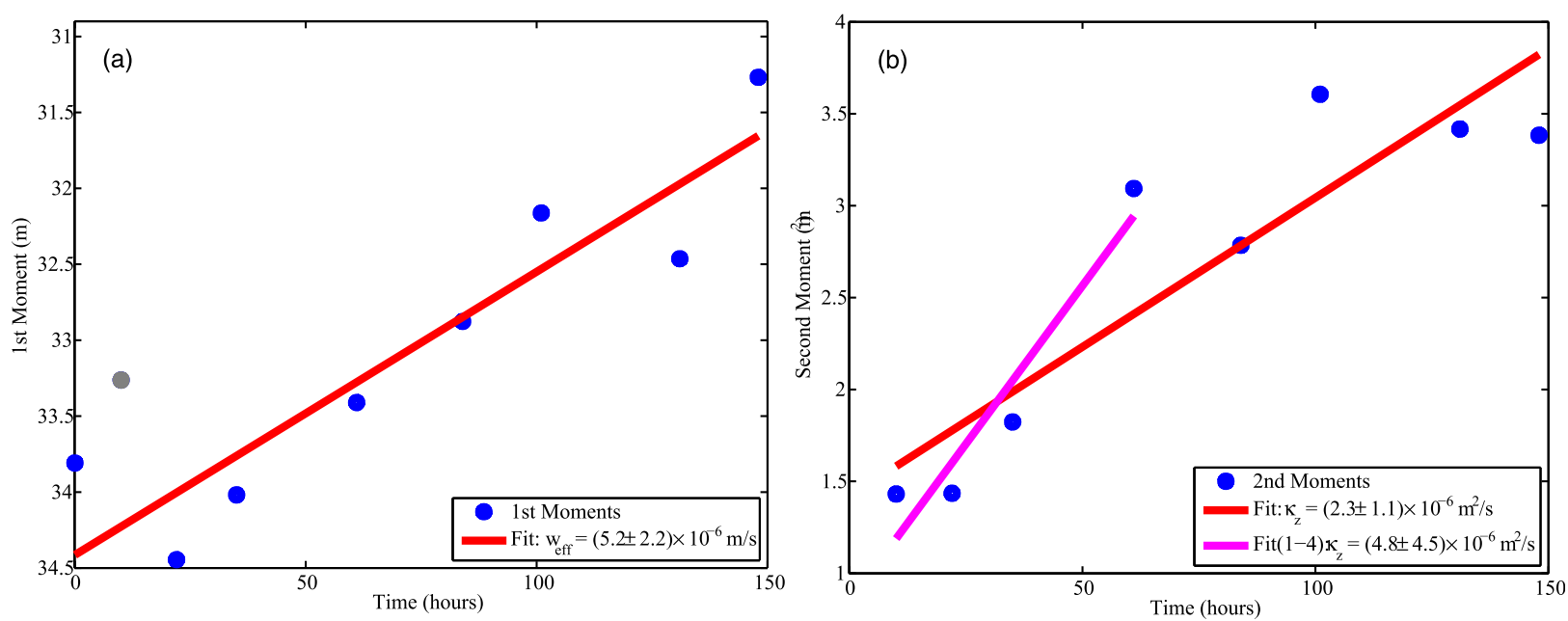

FIG. 15. Time evolution of (a) center of mass and (b) second moment in $z$ of dye distribution for the second rhodamine dye experiment. Bold red lines are linear fits to the data, with $95 \%$ confidence intervals of inferred vertical velocity $w_{\text {eff }}$ and diapycnal diffusivity $\kappa_{z}$ given in the legends. As in Fig. 8, the gray data point in (a) is from the first survey conducted using the OSU MVP, which was not used in the regression for $w_{\text {eff }}$, but was used in (b) for the regression for $\kappa_{z}$ (see text for explanation). Conversely, moments for the dye injection streak $(t=0)$ were used in (a), but not in (b) (again, see text for explanation). In (b), two fits were made, the first using the full dataset, the second using only the first four surveys. 

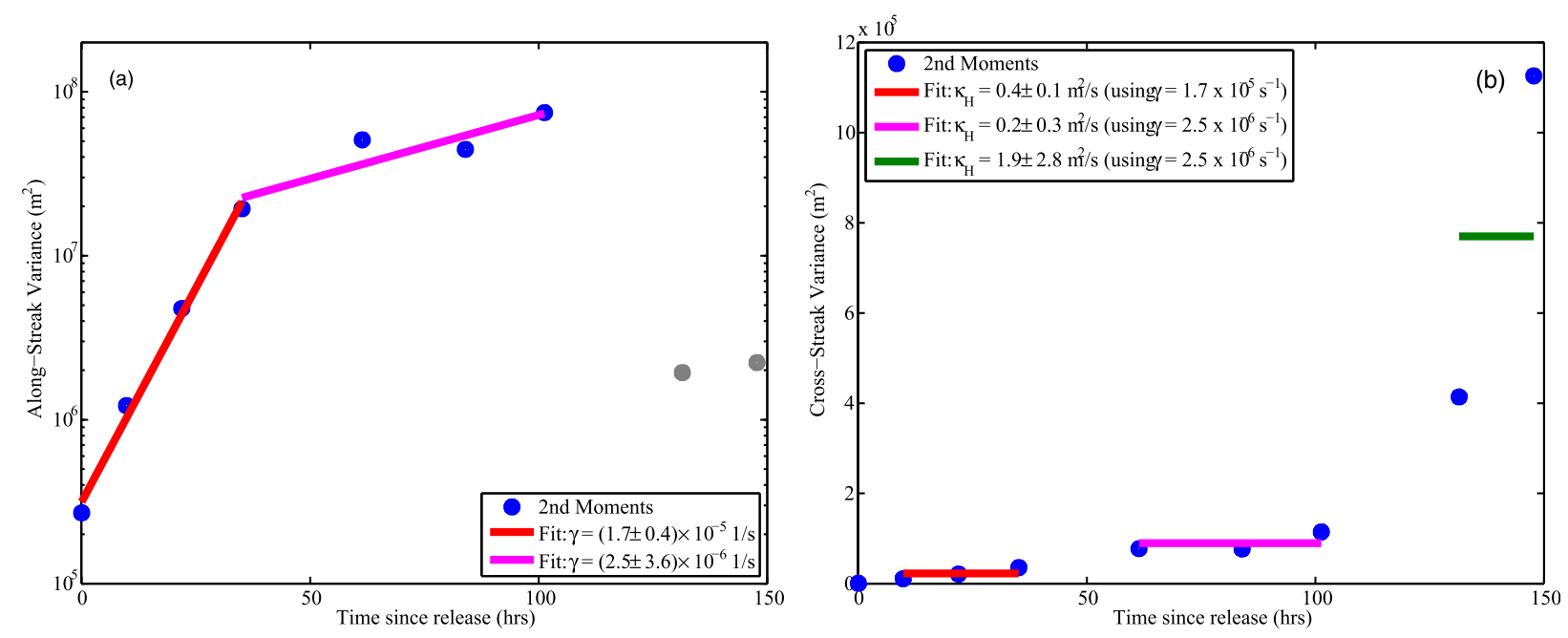

FIG. 16. Time evolution of the second moment in (a) the along-streak and (b) the cross-streak direction for second rhodamine dye experiment. In (a), two fits were made, the first using dye surveys from days $0-2$, the second using surveys from days $2-4$. In (b), estimates of $\kappa_{H}$ inferred from a balance between convergent strain rate and cross-streak isopycnal diffusivity per (8) are indicated in the legend and described further in the text.

time scale of order $1 / \gamma$, which in this case is order $1-$ 5 days, we use the strain rate inferred from the alongstreak growth early versus late in the experiment together with the observed streak width to estimate the small-scale diffusivity acting on the patch. Per (8), we again obtain multiple diffusivity estimates, $\kappa_{H}=0.4$ $\pm 0.1,0.2 \pm 0.3$, and $1.9 \pm 2.8 \mathrm{~m}^{2} \mathrm{~s}^{-1}$. Here the three estimates are based, respectively, on cross-streak moments estimated from surveys $1-3$ and the first strain rate estimated in Fig. 16a, and cross-streak moments estimated separately from surveys $4-6$ and $7-8$, both together with the second strain rate estimated in Fig. 16a. Again, we consider no single value of diffusivity to be a better or worse estimate, but rather take the range of estimates to represent likely upper and lower bounds of the true value. A more refined estimate of $\kappa_{H}$ that takes into account temporal variability of the observed growth in the dye moments as well as the integrated effects of vertical shear on the tilt of the patch is ongoing (D. A. Birch et al. 2020, unpublished manuscript), and again gives roughly similar results, that is, also of order $\kappa_{H} \sim$ $O(1) \mathrm{m}^{2} \mathrm{~s}^{-1}$.

\section{Discussion}

\section{a. Comparison of sites I and II results}

A notable finding from the two dye experiments presented here is the similarity of their diapycnal and isopycnal diffusivities despite the different conditions at the two sites. Specifically, both experiments yielded diapycnal diffusivities of order $\kappa_{z}=2-5 \times 10^{-6} \mathrm{~m}^{2} \mathrm{~s}^{-1}$ and isopycnal diffusivities of order $\kappa_{H}=0.2-3.0 \mathrm{~m}^{2} \mathrm{~s}^{-1}$, despite the second dye patch being embedded in a lateral strain rate that was nearly an order of magnitude larger for half of that experiment, and which stretched it along a temperature/salinity front to more than 5 times the length of the first patch. Rossby number [Ro $=$ $(\partial v / \partial x-\partial u / \partial y) / f]$, also varied by an order of magnitude between the two experiments, with Ro estimated from cross-frontal shipboard ADCP surveys reaching 0.1-0.3 at Site 1, and 1.0-1.5 at Site 2. Ro estimated from drogued drifter observations were somewhat smaller, ranging from 0.01 to 0.2 for Site 1 and from 0.2 to 0.4 for Site 2 , but still $\ll 1$ and approaching $O(1)$ for the two experiments, respectively. The difference between the ADCP and drifter-derived Ro estimates is likely due to the fact that the drifters moved slightly outside of the main dye patches, probably due to a combination of windage and mixed layer shear acting on the drogue tethers.

The fact that neither the diapycnal nor isopycnal diffusivity varied with differing strain rate or Ro between the two experiments is perhaps surprising. Previous work has shown that frontogenesis driven by mesoscale strain can lead to ageostrophic circulations that further enhance submesoscale fronts. As a front strengthens, meandering and eddy generation by baroclinic instability increases, countering isopycnal steepening caused by the mesoscale strain. Under certain conditions, instabilities along the front may become suppressed by the mesoscale strain (Spall 1997). However, more recent findings also show that as the cross-frontal scale decreases, the growth rate of the most unstable 
mode increases even faster (McWilliams and Molemaker 2011). For surface mixed layer fronts a combination of barotropic and baroclinic instabilities can thus lead to the arrest of frontogenesis at larger crossfrontal scales than previously thought, although many details of the underlying dynamics are still poorly understood (e.g., McWilliams 2016; Sullivan and McWilliams 2018).

The above in mind, and despite the growing body of literature that suggests that classic frontogenesis leads to increasingly ageostrophic dynamics, including increased small-scale horizontal and vertical turbulent eddy fluxes, we did not see a clear signal of increased small-scale diffusivities (i.e., within the scale of our dye patch) at Site 2 compared to Site 1 . This is despite the presence of a stronger front at Site 2. Further considering the ageostrophic secondary circulation associated with frontogenesis, this typically occurs on scales comparable to the front itself. While such circulation can readily lead to vertical velocities of order 10's of meters per day, on the time and space scales of our dye patches we would expect to see such motions as advective fluxes rather than diffusive fluxes. An analysis of secondary circulation within our dye patches is beyond the scope of the present analysis. However, for such advective fluxes to be diapycnal fluxes, they would have to have been accompanied by small-scale eddy diffusive processes acting within the front, which again would have shown up in our diffusivity estimates. Summarizing the above, despite increased strain, Rossby number, and also available potential energy associated with stronger lateral density gradients in the Site 2 experiment, we did not observe a concomitant increase in diapycnal or cross-frontal eddy diffusivity. Still unclear is whether this is due to straining in the cross-front direction limiting eddy scales, and hence eddy stirring, or whether it is an indication that the observed lateral mixing is not directly driven by frontal instability processes, but rather other processes such as internal waves; or whether it is for some other reason entirely.

Mechanisms aside, to within the uncertainty of the respective measurements, diapycnal diffusivities inferred from the dye were consistent with the turbulent diffusivity of temperature estimated from concurrent measurements of the dissipation of temperature variance by a swarm of EM-APEX floats by Lien et al. (2016). Our estimates of $\kappa_{H}$ are also consistent with results from previous dye and tracer experiments performed in a variety of settings, including over the continental shelf (Sundermeyer and Ledwell 2001), and within the pycnocline of the North Atlantic subtropical gyre (Ledwell et al. 1993, 1998), this despite the even lower horizontal strain rates $(\gamma=2-4 \times$ $10^{-6} \mathrm{~s}^{-1}$ and $\gamma=3 \times 10^{-6} \mathrm{~s}^{-1}$, respectively) observed in those two environments. Last, our isopycnal diffusivity estimates also fall within the range of scale dependent diffusivities reported by Okubo (1971), namely of $\sim 1 \mathrm{~m}^{2} \mathrm{~s}^{-1}$ at scales of order $1-5 \mathrm{~km}$. It is possible that both diapycnal and isopycnal diffusivity may be much larger in more energetic circumstances. However, the values we have found here seem to prevail within the pycnocline, and hence would apply, for example, to studies of nutrient transport on the time scale of a biogeochemical field study, though perhaps not for a whole season that includes storm events.

In the experiments described here, both dye patches remained mostly coherent/connected throughout most of the sampling period, with adjacent transects through the dye showing an overall cohesive patch, as opposed to many smaller-scale filaments and streaks. This general smoothness of the patches notwithstanding, local temporal and spatial variations in the dye distributions could also be seen. Two examples were a tail that developed at the eastern extremity of the Site I dye patch early in the experiment (see $45 \mathrm{~h}$ and later surveys in Fig. 5), which twisted from a northeastward to southeastward direction over the latter part of the experiment, and a filament that formed at the northernmost extent of the Site II patch. Within the first day or two after release, transects through the patch also showed distinct vertical shearing, reminiscent of previous experiments of this type performed over the New England continental shelf (Sundermeyer and Ledwell 2001). This shearing persisted throughout both experiments, eventually resulting in a large-scale tilt in the patch as it spread isopycnally. Also reminiscent of previous studies was evidence of small-scale patchiness within hours to a day after injection, primarily during the first experiment, suggestive of lateral stirring occurring on scales of order hundreds of meters to $1 \mathrm{~km}$. An example of a transect taken during the first dye experiment showing a tilted patch with two distinct dye concentration extrema is shown in Fig. 17.

Noteworthy in the second experiment was also the conspicuous absence of meanders or boluses at the scale of the dye patch width (i.e., a few kilometers) such as might be expected from frontal instability, despite the fact that the dye was embedded in an evolving front. Instead, the dye appeared to spread more or less diffusively on the scale of the patch width, showing little evidence of eddy stirring at these scales. The one exception to this was a filament observed at the northernmost extent of the patch during the final survey, which appears to have corresponded to the 

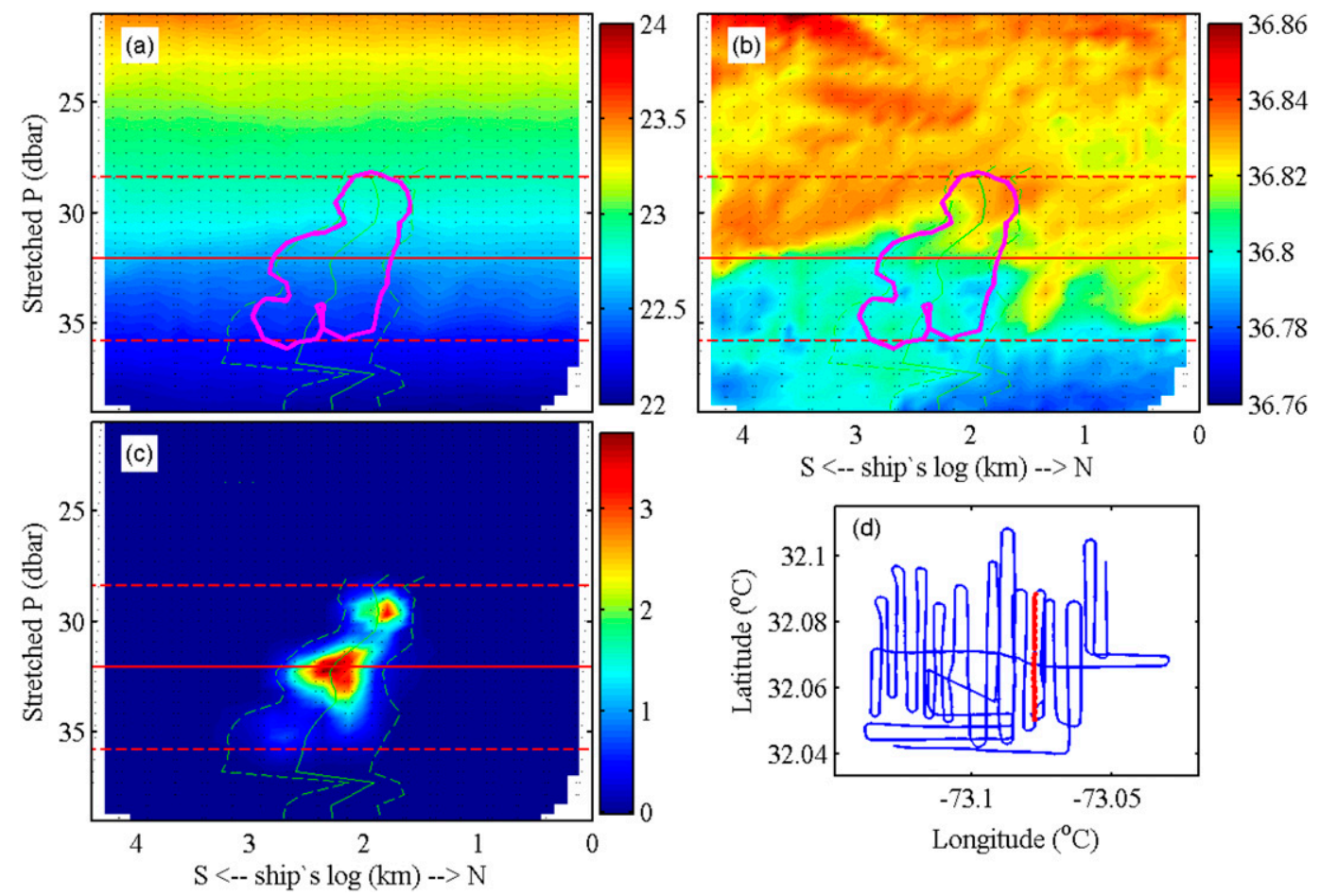

FIG. 17. Example of dye concentration measured during a single transect during the second survey of the first experiment showing a tilted patch with two distinct dye concentration extrema.

period of reduced strain rate at the northernmost reaches of the patch. Relating to our earlier discussion, whether the general absence of pronounced submesoscale frontal meanders was the result of the mesoscale strain rate inhibiting the growth of such features, or simply insufficient time for instabilities to reach finite amplitude and hence be detected is an area for further study.

\section{b. Notes on dispersion estimates}

Having estimated diapycnal and isopycnal diffusivities from the above observations, it is important to be clear exactly what these diffusivities do and do not represent. First, regarding diapycnal diffusivities, we have made every attempt to avoid bias in our estimates, first by ensuring that we delimited the patch during each survey, and second by sampling as uniformly as possible. This helped ensure we did not miss a particularly shallow or deep portion of the patch that may have been sheared away from the rest of the patch, and that our measured vertical profiles were thus representative of the true dye distributions. Further, we performed all gridding and averaging in isopycnal coordinates, allowing for diapycnal velocity and solar warming of the patch. However, an additional consideration is that the decrease of radiative heating with depth, which occurs naturally in seawater, causes diapycnal spreading of the dye that can be mistaken for diapycnal diffusion due to turbulence. Models including this effect indicate that our estimates of diapycnal diffusivity based on diapycnal spreading may overestimate that due to turbulent diffusion by about $25 \%$. That is, the diapycnal diffusivity due to turbulent mixing may be smaller than the $\kappa_{z}$ values we report here.

To compute isopycnal diffusivities, we explicitly accounted for the effects of the lateral strain rate, which elongated the patch in concert with diffusion in one direction, while opposing it in the other. We also computed lateral moments in isopycnal coordinates as a means of estimating true isopycnal diffusion, as opposed to a horizontal diapycnal/isopycnal hybrid, or depth-integrated diffusion (for further discussion of these distinctions see also Sundermeyer and Ledwell 2001). To make our estimates as robust as possible, we further averaged the second moments computed on all isopycnals within two standard deviations of the vertical center of mass of the dye patch before computing the time rate of change of the horizontal second moment. All horizontal moments were also computed normal to the major axis of the streak, allowing for curvature of the streak in the presence of a mesoscale strain rate-thus any larger-scale meanders in the along-streak axis of the patch were accounted for 
explicitly, and did not add to our cross-streak dispersion estimates.

\section{c. Clues to mechanisms}

Beyond our estimates of the effective diapycnal and isopycnal diffusivities from the two experiments, of significant interest is understanding the underlying dispersion mechanisms that drive them. The classical mechanism of internal wave shear dispersion discussed by Young et al. (1982) is being investigated at length in ongoing analysis (D. A. Birch et al. 2020, unpublished manuscript). Other candidate mechanisms are also explored to various degrees in other papers submitted to the LatMix special collection (https://journals.ametsoc.org/ topic/latmix) and as part of ongoing research. However, since no one process currently stands out as the dominant dispersion mechanism, rather than provide an exhaustive analysis for or against any one mechanism, we instead briefly consider distinguishing features of the observed dye patches and their evolution. This is done in the hopes of providing future studies a means of discriminating between candidate mechanisms.

While the processes controlling diapycnal mixing in the ocean are relatively well understood, the processes controlling isopycnal mixing, particularly at the submesoscale, are not. A key question is whether particular isopycnal mixing and/or stirring processes are consistent with the observations not only in terms of the resulting magnitude of the diffusion, but also in the character of the dye distributions they induce. Processes of interest include internal wave shear dispersion, dispersion by random internal waves, stirring by frontal instabilities and related submesoscale eddy processes, and vortical mode stirring.

Shear dispersion (e.g., Young et al. 1982) is fundamentally a diffusive process, with the effects of advection becoming apparent only in the vertical tilting/shearing of the patch. As noted above, vertical tilting/shearing of the dye was clearly observed in the present experiments. However, results presented by Shcherbina et al. (2015) as well as ongoing analysis (D. A. Birch et al. 2020, unpublished manuscript) suggest that, when combined with the observed diapycnal mixing, the resulting shear dispersion did not fully account for the observed lateral dispersion of the dye. Theoretical arguments by Kunze and Sundermeyer (2015) suggests that intermittency, the log-normality of turbulent dissipation, and the correlation between dissipation and shear variance may increase the effectiveness of shear dispersion beyond that predicted by Young et al. (1982), leading us to conclude that shear dispersion cannot yet be entirely ruled out. However, determining whether such enhanced shear dispersion effects can in fact explain the dye dispersion observed during LatMix is a subject of ongoing investigation.

Dispersion by random internal waves has also been postulated to lead to significant lateral dispersion, particularly in the presence of dissipation and nonlinear interactions associated with the forward energy cascade of a typical open ocean internal wave field (e.g., Bühler et al. 2013; Bühler and Guo 2016). For a canonical Garrett and Munk internal wave field the magnitude of such dispersion was predicted by Bühler and Guo (2016) to be of order $0.25 \mathrm{~m}^{2} \mathrm{~s}^{-1}$. This is similar to upper-bound estimates for internal wave shear dispersion estimated during LatMix (D. A. Birch et al. 2020, unpublished manuscript) and both of these are comparable to the smaller of the two lower-bound estimates obtained from the present analyses. We thus consider this process also still worthy of further investigation. Still unclear is whether there is a unique signature of dispersion by random internal waves that can be used observationally to distinguish it from other candidate mechanisms. Bühler et al. (2013) report that in their simulations particles exhibit "... inertial circles superimposed on a weak random walk." This suggests that a tracer patch undergoing dispersion by random internal waves may still appear diffusive in nature, similar to internal wave shear dispersion but without the requirement of diapycnal mixing and vertical shear to create the dispersion. However, again, further study is needed.

Yet another mechanism that can lead to isopycnal dispersion in the pycnocline is stirring by mixed layer frontal instabilities, the signature of which can extend below the mixed layer base into the pycnocline (e.g., Badin et al. 2011). In contrast to dispersion by random internal waves and shear dispersion, a typical signature of frontal instabilities is the straining and filamentation they create via differential advection. The resultant lateral stirring is widely recognized for its role in relaxing fronts (e.g., Thomas et al. 2008; Fox-Kemper et al. 2008; Molemaker et al. 2005; Taylor and Ferrari 2010). Frontal meanders and eddies typically have scales of order an appropriately defined baroclinic deformation radius, so that when viewed at these scales resultant tracer dispersion would more appropriately be described as stirring rather than a diffusive process. The Site I experiment was conducted in an area of relatively weak lateral density gradients, although the dye patch still exhibited a well-defined along- versus crossstreak orientation. However, for Site II we estimate the relevant near-surface baroclinic deformation radius as $R_{D}=M^{2} H / f^{2} \sim 2-3 \mathrm{~km}$, where $M^{2}$ is the lateral buoyancy gradient within the mixed layer, $H$ is the mixed layer depth, and $f$ is the local Coriolis frequency (e.g., Badin et al. 2011). Also per Badin et al. (2011), mixed 
layer eddies can penetrate the pycnocline to a depth of order $D=f R_{D} / N$, or $\sim 10-20 \mathrm{~m}$ based on parameters associated with the present experiments. This suggests that, at least for the second experiment, typical mixed layer eddies would have been comparable in size to the width of the dye patch, and would have penetrated deep enough to affect the dye. For Site I, differential advection at this scale could explain, for example, the northeast-to-southeast turning tail of the first patch noted previously. For Site II, meanders and instabilities at these scales should also have been evident had they formed. If they were present, they were not of largeenough amplitude to be readily detected. This notwithstanding, even large amplitude meanders at the scale of the patch would not account for the diffusive-like spreading of the dye within its $3-5-\mathrm{km}$ width unless the meanders were themselves immediately strained and homogenized, which still would have required significant small-scale cross-streak diffusion.

Finally, stirring by the vortical mode has also been investigated as a possible mechanism of isopycnal dispersion at scales from a few hundred meters to a few kilometers. Previous studies by Polzin and Ferrari (2004), Sundermeyer et al. (2005), and Sundermeyer and Lelong (2005) have argued that analytical and numerical predictions for dispersion by the vortical mode may be able to account for observed isopycnal diffusivities in the pycnocline of the North Atlantic subtropical gyre and over the New England continental shelf, respectively. However, direct observational evidence distinguishing contributions from vortical mode dispersion versus the other processes discussed above is still lacking. One distinguishing feature of dispersion by vortical mode is that at scales comparable to the vortical motions themselves dye dispersion is again expected to look more like stirring than mixing (e.g., see numerical simulations by Sundermeyer and Lelong 2005). This is consistent with results from the two dye experiments reported on here, which, like the ones reported on by Sundermeyer and Ledwell (2001), both show evidence of small-scale stirring early in their evolutions. Lien and Sanford (2019) used data from a swarm of EM-APEX floats deployed concurrently with the dye during LatMix to estimate vertical vorticity and vortex stretching, which they then separated into linear vortical mode and internal wave motions. They found that linear vortical mode contains from one to two orders of magnitude less energy than internal waves across a range of wavenumbers and frequencies. Note, however, that the slow but persistent motions associated with vortical mode can still lead to significant dispersion compared to the more energetic but primarily oscillatory internal wave motions. Quantitatively linking the results of
Lien and Sanford (2019) to observed isopycnal dispersion rates in LatMix remains a topic of ongoing investigation.

\section{d. Tracking of dye relative to $T$ and $S$ (spice) structure}

An aspect of the present observations that remains to be exploited is the rich temperature and salinity structure in which the dye patches were embedded. Independent of the dye, temperature and salinity distributions on their own provide information about the time and space scales of the underlying mixing. For example, Kunze et al. (2015) used salinity spectra along isopycnals to better understand what processes may or may not control spice variance at the submesoscale. Specifically, they found that salinity-gradient variance on isopycnals on $\sim 1-10-\mathrm{km}$ scales could be explained by internal-wave horizontal strain. Taking a more deterministic view, filaments of spice, or equivalently temperature and salinity along isopycnals, can be used along with estimates of along-isopycnal strain rate to infer effective lateral dispersion required to maintain the observed filament width. Examples for both the Site I and Site II experiments showing temperature and salinity filaments comparable in cross-streak scale to the fluorescent dye, are shown in Figs. 6 and 13 . Such observations support the idea that temperature and salinity, as natural tracer fields, contain comparable information about underlying processes as purposeful dye release experiments. An advantage of purposeful tracers, however, is the unambiguity of the marked fluid and hence tracking capabilities they provide.

\section{Summary and conclusions}

Two rhodamine dye experiments, each lasting approximately six days, were performed in early summer in the open ocean seasonal pycnocline in a region approximately $250 \mathrm{~km}$ southeast of Cape Hatteras, North Carolina. The study site was sufficiently east of the Gulf Stream to be considered typical of open ocean conditions. The first experiment was performed in a region of weak strain rate, of order $10^{-6} \mathrm{~s}^{-1}(0.01 f)$, the second in a region of intermediate strain rate, of order $10^{-5} \mathrm{~s}^{-1}$ (0.1f). Approximately daily surveys of the dye patch using a towed profiling sampling system were used to map the patches as they diffused and stretched vertically and horizontally, as well as diapycnally and isopycnally. Diapycnal diffusivities estimated from the dye ranged from $\kappa_{z}=2-5 \times 10^{-6} \mathrm{~m}^{2} \mathrm{~s}^{-1}$, while isopycnal diffusivities ranged from $\kappa_{H}=0.2-3.0 \mathrm{~m}^{2} \mathrm{~s}^{-1}$. Here the relatively larger spread in $\kappa_{H}$ was less the result of siteto-site variability, and more due to uncertainties in the 
background strain rate acting on the patch combined with uncertain time dependence.

Details of the dye distributions, together with measurements of velocity and temperature and salinity structure revealed a complicated story. The Site I (low strain) experiment exhibited minimal stretching, elongating to approximately 2.5 times its width over 6 days, but with a notable vertical tilt in the meridional direction. Early surveys of the dye showed patchy distributions indicative of small-scale stirring at scales of order a few hundred meters. Later surveys showed a mostly smooth, coherent patch more typical of a diffusive rather than stirring process at the scales of the now larger $5-10-\mathrm{km}$ patch. By contrast, the Site II (intermediate strain) experiment underwent significant stretching, advecting more than $150 \mathrm{~km}$ and elongating to more than $50 \mathrm{~km}$ in length while maintaining a streak width of order $3-5 \mathrm{~km}$. Surveys of the Site II dye patch early after its release did not resolve small scale variability indicative of stirring, and later surveys again showed a relatively smooth, coherent patch. Notably, the elongated streak also showed no evidence of large amplitude submesoscale meanders as might be expected due to baroclinic instability along a near-surface density front, save a single filament event at the northernmost extreme of the patch toward the end of the experiment. Whether such instabilities were simply not present, were suppressed by the mesoscale strain rate, or for some other reason were not manifest in the dye distributions is not clear, but warrants further study.

While much effort has been devoted in the field and via numerical and theoretical studies to understand the processes underlying observed submesoscale lateral dispersion (e.g., see other papers included in the LatMix special collection; https://journals.ametsoc.org/ topic/latmix), considerable effort is still required to isolate specific mechanisms. Lacking a clear mechanism, and hence parameterization, we are hard pressed to prescribe new recipes for submesoscale-resolving or submesoscale-permitting numerical models. However, in light of the present results, ongoing analyses and assessment of existing parameterizations should take into account not only the magnitude of the diffusivities, but also the character of the dye distributions, including early- versus late-time patchiness, the presence versus lack of frontal meanders (particularly for Site II), the vertical shearing/tilting of the patches, and any other relevant environmental variables and factors suspected of driving the dynamics. Some of these factors can be addressed via ancillary measurements in and around the dye patch, such as ADCP velocity profile measurements to quantify the integrated effect of vertical shear, or larger-scale surveys to assess lateral and vertical density gradients that may drive frontal instabilities. Others, such as degree of patchiness, require further research to better understand how small-scale variability within a dye patch relates to the specific stirring processes driving the dispersion.

Acknowledgments. Funding for the overall LatMix DRI was provided through the Office of Naval Research. Field work and analysis related to the dye and drifter experiments was supported jointly by the Office of Naval Research (Grants N00014-09-1-0194 and N0001415-1-2458 at UMassD, N00014-09-1-0175 at WHOI, and N00014-09-1-0460 at OSU) and the National Science Foundation Physical Oceanography Program (Grants OCE-0751734 at UMassD, and OCE-0751653 at WHOI). A portion of the field instrumentation used in this project was purchased under DURIP Grant N00014-09-1-0825. The authors also thank the able captain and crew of R/V Cape Hatteras, and the at sea support of the entire LatMix DRI group, including A.-M. Brunner-Suzuki, D. Debiegun, M.-P. Lelong, and G. Badin, who all assisted at various times with the dye and drogued drifters. Shipboard data for the LatMix field experiments are available via the R2R Repository (http:// www.rvdata.us/). Data from towed hydrographic and dye sensors are available at M. Sundermeyer's website (http:// www.smast.umassd.edu/mixing/lidar_ONR_NSF.php). In memoriam of Murray Levine.

\section{REFERENCES}

Badin, G., A. Tandon, and A. Mahadevan, 2011: Lateral mixing in the pycnocline by baroclinic mixed layer eddies. J. Phys. Oceanogr., 41, 2080-2101, https://doi.org/10.1175/JPO-D-11-05.1.

Bühler, O., and Y. Guo, 2016: Particle dispersion by nonlinearly damped random waves. J. Fluid Mech., 786, 332-347, https:// doi.org/10.1017/jfm.2015.605.

— N. Nrisouard, and M. Holmes-Cerfon, 2013: Strong particle dispersion by weakly dissipative random internal waves. J. Fluid Mech., 719, R4, https://doi.org/10.1017/jfm.2013.71.

Capet, X., J. C. McWilliams, M. J. Molemaker, and A. F. Shchepetkin, 2008a: Mesoscale to submesoscale transition in the California Current System. Part I: Flow structure, eddy flux, and observational tests. J. Phys. Oceanogr., 38, 29-43, https://doi.org/10.1175/2007JPO3671.1.

,,-- - $\longrightarrow$, and 2008b: Mesoscale to submesoscale transition in the California Current System. Part III: Energy balance and flux. J. Phys. Oceanogr., 38, 2256-2269, https:// doi.org/10.1175/2008JPO3810.1.

D'Asaro, E., C. Lee, L. Rainville, R. Harcourt, and L. Thomas, 2011: Enhanced turbulence and energy dissipation at ocean fronts. Science, 332, 318-322, https://doi.org/10.1126/science.1201515.

Fox-Kemper, B., R. Ferrari, and R. Hallberg, 2008: Parameterization of mixed layer eddies. Part I: Theory and diagnosis. J. Phys. Oceanogr., 38, 1145-1165, https://doi.org/10.1175/2007JPO3792.1.

Hoskins, B. J., and F. P. Bretherton, 1972: Atmospheric frontogenesis models: Mathematical formulation and solution. 
J. Atmos. Sci., 29, 11-37, https://doi.org/10.1175/15200469(1972)029<0011:AFMMFA > 2.0.CO;2.

Kunze, E., and M. A. Sundermeyer, 2015: The role of intermittency in internal-wave shear dispersion. J. Phys. Oceanogr., 45 2979-2990, https://doi.org/10.1175/JPO-D-14-0134.1.

—, J. Klymak, R.-C. Lien, R. Ferrari, C. Lee, M. Sundermeyer, and L. Goodman, 2015: Submesoscale water-mass spectra in the Sargasso Sea. J. Phys. Oceanogr., 45, 1325-1338, https:// doi.org/10.1175/JPO-D-14-0108.1.

Ledwell, J. R., A. J. Watson, and C. S. Law, 1993: Evidence for slow mixing across the pycnocline from an open ocean tracer release experiment. Nature, 364, 701-703, https://doi.org/ 10.1038/364701a0.

,-- , and -1998 : Mixing of a tracer in the pycnocline. J. Geophys. Res., 103, 21 499-21 529, https://doi.org/10.1029/ 98JC01738.

Lévy, M., R. Ferrari, P. J. Franks, A. P. Martin, and P. Rivière, 2012: Bringing physics to life at the submesoscale. Geophys. Res. Lett., 39, L14602, https://doi.org/10.1029/2012GL052756.

Lien, R.-C., and T. B. Sanford, 2019: Small-scale potential vorticity in the upper ocean thermocline. J. Phys. Oceanogr., 49, 18451872, https://doi.org/10.1175/JPO-D-18-0052.1.

$\longrightarrow,-$ J. A. Carlson, and J. H. Dunlap, 2016: Autonomous microstructure EM-APEX floats. Methods Oceanogr., 17, 282-295, https://doi.org/10.1016/j.mio.2016.09.003.

Lin, J. T., and Y. H. Pao, 1979: Wakes in stratified fluids. Annu. Rev. Fluid Mech., 11, 317-338, https://doi.org/10.1146/ annurev.fl.11.010179.001533.

Mahadevan, A., 2006: Modeling vertical motion at ocean fronts: Are nonhydrostatic effects relevant at submesoscales? Ocean Modell. 14, 222-240, https://doi.org/10.1016/j.ocemod.2006.05.005.

- , and D. Archer, 2000: Modeling the impact of fronts and mesoscale circulation on the nutrient supply and biogeochemistry of the upper ocean. J. Geophys. Res., 105, 12091225, https://doi.org/10.1029/1999JC900216.

_ mesoscale vertical motion at ocean fronts. Ocean Modell., 14, 241-256, https://doi.org/10.1016/j.ocemod.2006.05.006.

— A. Tagliabue, L. Bopp, A. Lenton, L. Mémery, and M. Lévy, 2011: Impact of episodic vertical fluxes on sea surface $\mathrm{pCO}_{2}$. Philos. Trans. Roy. Soc. London, 369A, 2009-2025, https:// doi.org/10.1098/rsta.2010.0340.

_- A. Tandon, and R. Ferrari, 2010: Rapid changes in mixed layer stratification driven by submesoscale instabilities and winds. J. Geophys. Res., 115, C03017, https://doi.org/10.1029/ 2008JC005203.

Martin, A. P., 2003: Phytoplankton patchiness: The role of lateral stirring and mixing. Prog. Oceanogr., 57, 125-174, https:// doi.org/10.1016/S0079-6611(03)00085-5.

McWilliams, J. C., 2016: Submesoscale currents in the ocean. Proc. Roy. Soc. London, 472A, 20160117, https://doi.org/10.1098/ rspa.2016.0117.

__ , and M. J. Molemaker, 2011: Baroclinic frontal arrest: A sequel to unstable frontogenesis. J. Phys. Oceanogr., 41, 601619, https://doi.org/10.1175/2010JPO4493.1.
Molemaker, M. J., J. C. McWilliams, and I. Yavneh, 2005: Baroclinic instability and loss of balance. J. Phys. Oceanogr., 35, 1505-1517, https://doi.org/10.1175/JPO2770.1.

,-- , and X. Capet, 2010: Balanced and unbalanced routes to dissipation in an equilibrated eady flow. J. Fluid Mech., 654, 35-63, https://doi.org/10.1017/S0022112009993272.

Okubo, A., 1971: Oceanic diffusion diagrams. Deep-Sea Res., 18, 789-802, https://doi.org/10.1016/0011-7471(71)90046-5.

Polzin, K. L., and R. Ferrari, 2004: Isopycnal dispersion in NATRE. J. Phys. Oceanogr., 34, 247-257, https://doi.org/10.1175/ 1520-0485(2004)034<0247:IDIN > 2.0.CO;2.

Richardson, L. F., 1926: Atmospheric diffusion shown on a distance-neighbour graph. Proc. Roy. Soc. London, 110A, 709-737, https://doi.org/10.1098/rspa.1926.0043.

Shcherbina, A. Y., and Coauthors, 2015: The LatMix summer campaign: Submesoscale stirring in the upper ocean. Bull. Amer. Meteor. Soc., 96, 1257-1279, https://doi.org/10.1175/ BAMS-D-14-00015.1.

Smart, P. L., and I. M. S. Laidlaw, 1977: An evaluation of some fluorescent dyes for water tracing. Water Resour. Res., 13, 1533, https://doi.org/10.1029/WR013i001p00015.

Spall, M. A., 1997: Baroclinic jets in confluent flow. J. Phys. Oceanogr., 27, 1054-1071, https://doi.org/10.1175/1520-0485(1997)027<1054: $\mathrm{BJICF}>2.0 . \mathrm{CO} ; 2$.

Sullivan, P. P., and J. C. McWilliams, 2018: Frontogenesis and frontal arrest of a dense filament in the oceanic surface boundary layer. J. Fluid Mech., 837, 341-380, https://doi.org/ 10.1017/jfm.2017.833.

Sundermeyer, M. A., and J. R. Ledwell, 2001: Lateral dispersion over the continental shelf: Analysis of dye release experiments. J. Geophys. Res., 106, 9603-9621, https://doi.org/ 10.1029/2000JC900138.

, and M.-P. Lelong, 2005: Numerical simulations of lateral dispersion by the relaxation of diapycnal mixing events. J. Phys. Oceanogr., 35, 2368-2386, https://doi.org/10.1175/ JPO2834.1.

, J. R. Ledwell, N. S. Oakey, and B. J. W. Greenan, 2005: Stirring by small-scale vortices caused by patchy mixing. J. Phys. Oceanogr., 35, 1245-1262, https://doi.org/10.1175/ JPO2713.1.

Taylor, J. R., and R. Ferrari, 2010: Buoyancy and wind-driven convection at mixed layer density fronts. J. Phys. Oceanogr., 40, 1222-1242, https://doi.org/10.1175/2010JPO4365.1.

Thomas, L. N., A. Tandon, and A. Mahadevan, 2008: Submesoscale processes and dynamics. Ocean Modeling in an Eddying Regime, Geophys. Monogr., Vol. 177, Amer. Geophys. Union, 17-38, https://doi.org/10.1029/177GM04.

Wilson, J. F., Jr., E. D. Cobb, and F. A. Kilpatrick, 1984: Fluorometric procedures for dye tracing. U. S. Geological Survey Tech. Rep. 84-234, 53 pp., https://pubs.usgs.gov/of/ 1984/0234/report.pdf.

Young, W. R., P. B. Rhines, and C. J. R. Garrett, 1982: Shear-flow dispersion, internal waves and horizontal mixing in the ocean. J. Phys. Oceanogr., 12, 515-527, https://doi.org/10.1175/15200485(1982)012<0515:SFDIWA $>2.0$. CO;2. 